\title{
Synoptic Pattern Analysis and Climatology of Ice and Snowstorms in the Southern Great Plains, 1993-20110
}

\author{
ESTHER D. MULLENS \\ South Central Climate Science Center, Norman, Oklahoma \\ LANCE M. Leslie AND Peter J. LAMB ${ }^{\mathrm{a}}$ \\ Cooperative Institute for Mesoscale Meteorological Studies, and School of Meteorology, \\ University of Oklahoma, Norman, Oklahoma
}

(Manuscript received 10 December 2015, in final form 11 April 2016)

\begin{abstract}
Winter storms in the southern United States can significantly impact infrastructure and the economy. In this study, National Centers for Environmental Information Storm Event Database and local climate summaries, are used to develop a spatial climatology of freezing precipitation (freezing rain and ice pellets) and snow over the southern Great Plains, 1993-2011. Principal component analysis is performed on the 500-hPa height field, at the approximate onset time of precipitation, for 33 freezing precipitation and 42 snow case studies, to differentiate common synoptic flow fields associated with precipitation type. The five leading patterns for each precipitation type are retained. Composites of temperature, moisture, pressure, and wind fields are constructed and extended $24 \mathrm{~h}$ before and after precipitation initiation to track the storm system evolution. Many 500-hPa flow fields are similar for both precipitation types. However, snow-dominant events have stronger and/or more frequent surface cyclone development. Freezing precipitation is associated with the southward propagation of an Arctic anticyclone well ahead of precipitation, weak or absent surface cyclone formation, and a more western trough axis. High-impact ice storms in the region often have slow-moving upper-level flow, persistent isentropic ascent over a surface quasi-stationary front with strongly positive moisture anomalies, and warm layer airmass trajectories originating over the Gulf of Mexico. The results here are based on a relatively small sample size. However, this work is intended to be useful for forecasters, in particular as a pattern recognition aid in predicting the evolution of precipitation within southern Great Plains winter storms.
\end{abstract}

\section{Introduction}

The southern Great Plains of the United States (hereafter SGP; Fig. 1) experience a variety of winter weather. Winter precipitation events, including snow, freezing rain, and ice pellets (Changnon et al. 2006), typically are infrequent, but the socioeconomic impacts are particularly adverse, because of the limited winter weather infrastructure and adaptive capacity (e.g.,

\footnotetext{
${ }^{\text {a }}$ Deceased.

Supplemental information related to this paper is available at the Journals Online website: http://dx.doi.org/10.1175/WAF-D-150172.s1.
}

Corresponding author address: Esther D. Mullens, Ste. 2100, 201 Stephenson Pkwy., Norman, OK 73019.

E-mail: esther.white@ou.edu
Changnon 2003). An Oklahoma-specific study by Grout et al. (2012) noted seven federal disaster declarations, resulting in over $\$ 500$ million in aid expenditures, for winter storms between 2000 and 2010. A county-level warning-type analysis established that most of the aid was allocated to counties experiencing ice storms that produced prolonged or heavy freezing rain in excess of 0.25 in. [National Weather Service definition; e.g., Grout et al. (2012)]. Common impacts included disruption of transportation and infrastructure, heightened personal injury risk, and power-line damage from ice loading (Rauber et al. 2001; Ressler et al. 2012). An economic analysis of ice storm impacts by Changnon (2003) ranked the south-central region fourth in the nation in total damage loss (1949-2000), but with the lowest number of freezing rain days (3.5) necessary to exceed $\$ 1$ million in financial losses. This result was attributed to infrequent, but comparatively high, ice accumulations 

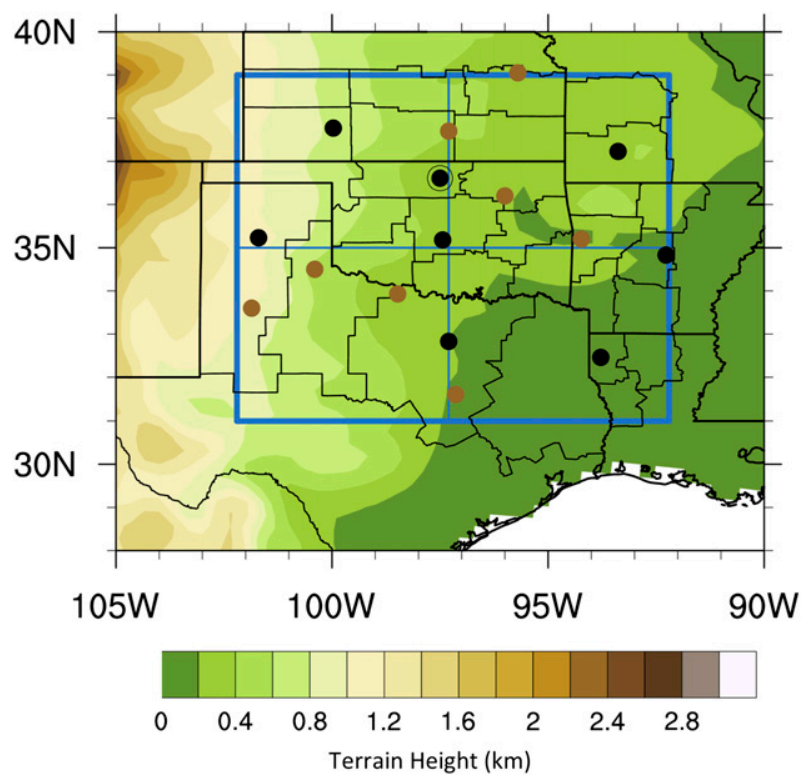

FIG. 1. Map of the SGP domain, including the study region (blue box) and its quadrants (thin blue lines); NOAA CDs used (thin black lines); rawinsonde stations (black dots) with the ACRF Lamont, OK, site circled; additional station sites used in estimating accumulation/liquid water equivalent (LWE) (brown dots); and elevation above sea level (shaded; in $\mathrm{km}$ ). Station sites include Amarillo, TX; Childress, TX; Dallas-Fort Worth, TX; Dodge City, KS; Fort Smith, AR; Lamont; Little Rock, AR; Lubbock, TX; Oklahoma City, OK; Shreveport, LA; Springfield, MO; Topeka, KS; Tulsa, OK; Waco, TX; Wichita Falls, TX; and Wichita, KS.

associated with moisture-abundant, long single-duration events.

Despite the aforementioned studies, less is known of the regional climatology and the synoptic and mesoscale evolution of winter storms for the SGP. Further detailed analysis is necessary, as event infrequency allows limited opportunities for forecasters to acquire predictive experience (e.g., Lackmann 2011, p. 221). This study seeks to develop the needed synoptic climatology, emphasizing storms associated with freezing precipitation (freezing rain, freezing drizzle, and ice pellets), with a focus on freezing rain.

While regional analyses remain few, national studies of freezing precipitation show that the SGP lies near the southwest edge of a relative maximum in freezing rain frequency, extending northeastward through Michigan and New England (e.g., Riehl et al. 1952; Bennett 1959; Baldwin 1973; Bernstein 2000; Rauber et al. 2000, 2001; Robbins and Cortinas 2002; Cortinas et al. 2004). Another maximum extends east of the Appalachians, northward from western North Carolina. Rawinsonde vertical profiles have been used to document characteristic thermodynamic structures, evolution, and microphysics accompanying different phases of freezing and frozen precipitation in those regions (e.g., Stewart 1985; Stewart and King 1987; Huffman and Norman 1988; Czys et al. 1996; Zerr 1997; Bernstein 2000; Robbins and Cortinas 2002). This approach has established typical thermal and microphysical bounds to precipitation types for automated precipitation typing of model and observational data.

For synoptic-scale surface features during freezing rain, Rauber et al. (2001) identified four (three) nonterrain (Appalachian) synoptic archetypes east of the Rocky Mountains. The nonterrain patterns include 1) an elongated zone parallel and north of a surface stationary front, within a shallow cold (arctic) air mass; 2 ) the warm front/occlusion sector of a surface cyclone; 3 ) the eastern sector of a cyclone traversing south of an Arctic anticyclone; and 4) the area west of an Arctic anticyclone. Robbins and Cortinas (2002) further elucidated the nature of the tropospheric thermodynamic environment for freezing rain from nationwide soundings, establishing the role of warm-air advection in developing and maintaining a warm layer, and as the primary mechanism for ascent north of surface quasi-stationary fronts. Ressler et al. (2012) analyzed synoptic features accompanying freezing rain in the St. Lawrence River valley, Canada, stratifying events on the proximity of the 500-hPa geopotential axis to the study domain. Proximal trough axes ("east composites") generally were associated with strong dynamic forcing, surface cyclone development, and short-duration heavy precipitation. In contrast, a trough axis well to the west typically produced larger low-level thickness anomalies, longerduration events, and lower precipitation rates.

The above research provides the context, framework, and basis used here to extend the study geographically to the SGP. A regional winter storm dataset is compiled for 1993-2011, which is used in two ways. First is to develop a climatology of freezing/frozen precipitation frequency and seasonal variability, as well as to evaluate the common thermodynamic environment. This latter aspect uses rawinsonde data extracted for different precipitation phases. Second, from principal component (PC) and composite analysis, five characteristic synoptic patterns conducive to the formation of widespread freezing rain ("ice") and snow are identified for the domain, and emphasis is placed on differences in the evolution of pressure, wind fields, moisture, and temperature that may aid regional forecasters in pattern recognition and understanding of regional precursors and characteristics for significant winter weather.

\section{Data and methods}

\section{a. Dataset development}

The SGP study region is shown in Fig. 1. It extends across approximately $10^{\circ}\left(8^{\circ}\right)$ of longitude (latitude), 
including Oklahoma and portions of five adjacent states. To identify and partition winter storm events broadly by location of impact, the domain was subdivided into four quadrants. A consistent identification and evaluation of winter storms required data from multiple sources.

First, a dataset for winter storm events from January 1993 to February 2011 was compiled using the NOAA/ National Centers for Environmental Information (NCEI) Storm Events Database (http://www.ncdc.noaa. gov/stormevents/) and the Storm Data publication. This database archives notable weather events by county, state, and approximate duration, providing brief descriptions of salient features. The first year (1993) of the present analysis coincided with the start year of the online record when this research was initiated. Information concerning winter storm type (ice storm, freezing rain and drizzle, ice pellets, snow) was gathered initially by state and, then, manually combined to form a spreadsheet dataset including onset date and time, duration, quadrant and county locations, and precipitation phase type for each event traversing the domain. Here, thresholds for retention included peak snow accumulations from each storm description exceeding 3 in. and/or freezing rain accumulations exceeding $0.1 \mathrm{in}$. The extent, or precise location, of these accumulations was not evaluated, owing to limited spatial data. Approximately 150 winter weather events were identified. To be classed as a separate storm system typically required a temporal gap of $48 \mathrm{~h}$ between event start times at a given location. For a few long-duration winter precipitation episodes, events were considered as distinct where breaks in precipitation of at least $12 \mathrm{~h}$ were observed. One such case is included in section $3 b$ (Table A1) but its inclusion or removal was not found to highly impact our results (not shown).

Second, because information from the Storm Events/ Storm Data archive is highly descriptive, locations of precipitation and attendant phase types were further constrained by NCEP surface analysis charts at 3-h intervals (http://nomads.ncdc.noaa.gov/ncep/NCEP), and from local station climate summaries (sourced from http://www.ncdc.noaa.gov/IPS/lcd/lcd.html), the locations of which are displayed in Fig. 1. Events after 2005 could be additionally documented using regional surface analyses at 1-h intervals, available from the Meteorological Case Study Selection database (http://www2. mmm.ucar.edu/imagearchive/). The spatial maps were used primarily to visually determine the climate divisions (CDs) impacted by each event in the database. Unfortunately, no direct, spatially gridded, observations of freezing precipitation accumulation were available (e.g., Ressler et al. 2012). The North American Regional Reanalysis (NARR; Mesinger et al. 2006) provides estimates of ice pellets, freezing rain, and snow, via a categorical fractional variable (1/0), supplied at $3-\mathrm{h}$ intervals, which was used to approximate locations in addition to in situ observations and analysis charts. Work by Blunden and Arndt (2011) determined that categorical freezing rain reliably reproduced the seasonal climatology for multiple station sites. Nonetheless, this parameter has not been comprehensively verified and, so, was applied alongside at least one other observation.

The availability of county-scale information from Storm Data, and spatial information from NWS stations, along with NCEI charts, was used to determine monthly and annual ice and snow frequencies for the NOAA CDs delineated in Fig. 1. The method used to determine these frequencies for each $\mathrm{CD}$ is described in Mullens (2014, chapter 2 and its appendix). Subsequently, a domain-wide climatology of winter storm events was constructed from the number of days of freezing precipitation (any combination of freezing rain, freezing drizzle, ice pellets) or snow, annually within each CD. This value was scaled by the mean area of all CDs evaluated. The mean area has a scaling factor of 1 , and the ice and snow counts in each CD were adjusted up or down depending on the their size relative to the mean. Values were expressed as the (scaled) average number of days per year for 1993-2011. Despite removing higher-resolution detail, the approach captures the gross ice and snow distributions, and the multiobservation assessment likely mitigated potential reporting inconsistencies in the county data (e.g., Branick 1997; Mullens 2014). Given the limitations of the aforementioned data sources in measuring the duration and intensity of precipitation, no attempt was made to differentiate events in those respects. Thus, short- and long-duration events within a 24-h (ending 0000 UTC) period are weighted equally (i.e., 1 day). Furthermore, separation of ice pellet observations from freezing rain and freezing drizzle is highly difficult for the spatial analysis, particularly as these phase types can occur concurrently, and was outside the scope of this work.

An additional analysis briefly examined the surface and tropospheric thermal and wind profiles by precipitation phase. Rawinsonde soundings were obtained from the University of Wyoming sounding archive (http://weather.uwyo.edu/upperair/sounding.html), for stations shown in Fig. 1, launched within $1 \mathrm{~h}$ of a winter precipitation observation for that station site. Seven stations are members of the National Weather Service network, with soundings every $12 \mathrm{~h}$ (0000 and 1200 UTC). The remaining location, operated by the ARM Climate Research Facility (ACRF; Stokes and Schwartz 
1994; Ackerman and Stokes 2003), provided research soundings every $6 \mathrm{~h}(0000,0600,1200$, and $1800 \mathrm{UTC})$, which were accessed from the ARM data archive (http:// www.archive.arm.gov/armlogin/login.jsp). Corresponding precipitation types were largely derived from surface NCEI weather summaries for this site. In total, 97 soundings for freezing precipitation were considered (57 freezing rain, 19 ice pellets, and 21 freezing drizzle), along with 24 snowfall profiles. The sample was constrained by a small spatial and temporal domain, the requirement for temporal and spatial collocation of precipitation type with sounding profiles (e.g., Rauber et al. 2001), and the relatively low event frequency.

\section{b. Synoptic pattern analysis}

To characterize the evolution of winter storms based on phase type, storms from the above total event dataset were subjectively (manually) categorized as ice or "snow" depending on whether they exhibited a broad and well-defined region of accumulating freezing rain. Snow events were winter storms with snow accumulation exceeding $3 \mathrm{in}$. in at least one location, and a largely absent mixed freezing precipitation transition zone within the domain, meaning freezing rain was absent, or of only short duration and intensity (accumulations $<$ 0.1 in.). Where reported, ice pellet accumulations $>$ $0.1 \mathrm{in}$. were permitted as the presence/absence of freezing rain was of primary concern. For each ice event, a broad and well-defined mixed-phase region was observed, even if snow also occurred within the domain. In fact, it was often the case that ice events were accompanied by snow in other portions of the domain, or successive to a freezing rain event at a given region. Some events were difficult to categorize, since they exhibited both widespread heavy snow ( $>6 \mathrm{in}$.) and freezing rain $(>0.25 \mathrm{in}$.), with both precipitation types present at time of onset $T_{0}$. Since the dominant phase of these (five) high-impact events was ambiguous, they were assigned to both snow and ice categories. Inclusion of these events was desired, particularly for the ice category, since they included a significant mixed-phase component. Removing these "shared" events from the snow dataset did not substantially alter the patterns depicted in our results, and over $80 \%$ of member events to those patterns types were identical to that revealed in the appendix (see Table A2) (not shown). Excluded from the datasets were shortduration, low-accumulation events $(<6 \mathrm{~h})$, and lowaccumulation, mixed-phase events. Also, events with precipitation accumulation areas restricted to only one study region quadrant (Fig. 1) were removed, reducing the possibility of bias toward a specific subregion.

Totals of 42 snow and 33 freezing precipitation events were identified (tabulated in the appendix), and the instantaneous 500-hPa geopotential height field at $T_{0}$ for each event was placed in the snow or ice dataset, respectively. The $T_{0}$ value was estimated first from Storm Data reports for the impacted regions and, subsequently, from the other aforementioned resources. Nonetheless, given that it can be difficult to accurately determine when winter precipitation initiated across a broad region, it must be assumed that $T_{0}$ is inherently uncertain. To account for this uncertainty to some degree, three additional assumptions for event onset were employed. This included secular offsets to estimated $T_{0}$ by +6 and $-6 \mathrm{~h}$ (twice the temporal resolution of $3 \mathrm{~h}$ ), and a randomized 6-h offset, totaling four datasets for each precipitation-type category.

The $500-\mathrm{hPa}$ data were from the NARR, for a contiguous U.S. domain spanning $20^{\circ}-50^{\circ} \mathrm{N}$ and $140^{\circ}-80^{\circ} \mathrm{W}$. The NARR dataset is a higher-resolution extension of the NCEP-NCAR global reanalysis (Kalnay et al. 1996) for the North American region, developed using a 32-km grid-size NCEP Eta Model with a Regional Data Assimilation System (RDAS). For computational efficiency, NARR 500-hPa geopotential height was interpolated to $1^{\circ} \times 1^{\circ}$ horizontal grid spacing.

To isolate a range of event subgroups exhibiting similar geopotential evolutions over the contiguous U.S. domain, within the snow and ice hydrometeor categories, a mixture of principal component and composite analyses was employed. The initial categorization was determined via a promax (oblique) rotated principal component (PRPC) analysis (Richman 1986), performed separately for the snow and ice 500-hPa geopotential height datasets. This level was used since midtropospheric circulation strongly influences the development and strength of surface weather systems, as well as their associated thermodynamic evolution and movement. Past studies have regularly utilized 500-hPa geopotential height for weather regime classification (e.g., Blackmon 1976; Horel 1981; Casola and Wallace 2007). First, gridded snow and ice height fields over the contiguous U.S. domain were standardized and adjusted for latitude using a simple cosine weighting function. PRPCs of the standardized snow and ice fields were evaluated for this domain in "temporal mode" ( $T$ mode), for which the simple structure rotation identifies subgroups of cases in time when similar spatial patterns are observed (Richman 1986). This approach requires that the data matrix be ordered so that the variables refer to each discrete time point ("event"), which permits the synoptic-scale spatial distribution of the height field to be compared across events. The T-mode analysis is appropriate within this context, as the temporal dimension involves discrete events rather than continuous sequences, and the aim is to identify characteristic atmospheric flow fields. 
The initial number of unrotated PCs identified for possible retention and rotation was limited to those with adequate eigenvalue separation from the preceding PC (North et al. 1982). Final determination of the number of PRPCs to retain and analyze was made by successively reducing the number of rotated PCs from 10 to 2 , and evaluating the congruence between each PRPC vector and the temporal correlation matrix of the original variable. This treatment employed the congruence coefficient from Harman (1976), as applied by Richman and Lamb (1985, their appendix). The selection of PRPCs selected for further treatment (four for ice, and three for snow) had the highest average congruence coefficients among the 2-10 rotated sets and four $T_{0}$ assumptions, with the additional requirement that each PRPC congruence was $\geq 0.9$. If there was discrepancy between these parameters between $T_{0}$ and the three offset assumptions (e.g., in number of PRPCs to retain), the values for $T_{0}$ were prioritized.

For the two separate categories, events were binned into groups based on the magnitude $(\geq \pm 0.45)$ of the PRPC loading correlation for each event against each PRPC score (spatial pattern). The low correlation threshold balanced the need to preserve the adequate separation required to identify distinct $500-\mathrm{hPa}$ height fields, while retaining enough events to ensure the representativeness of the resulting composite fields. The events corresponding to each PRPC score were computed separately for $T_{0}$ and the offset assumptions. Final assignments of member events required the event to be present in the same event groupings for at least two of the four PRPCs for ice and snow, respectively. This approach provides a more robust determination of composite members, as it excludes events that are highly sensitive to the precise specification of $T_{0}$. This method updates the climatology shown by Mullens (2014).

Since events can be positively or negatively correlated over the above threshold with a given loading pattern, two pattern types may be identified from each PRPC score. Therefore, for snow (ice), the three (four) PRPCs produced up to six (eight) 500-hPa patterns, constructed by compositing the 500-hPa height fields for each event group. The representativeness of the pattern types was confirmed from the PRPC scores by using a Z-score conversion to geopotential height (e.g., Mullens 2014, chapter 3), which resulted in very similar pattern structure as revealed from compositing member events (see Figs. S1-S10 in the online supplement to this article). The evolution of each pattern was further clarified by compositing these same event groups for $24 \mathrm{~h}$ prior to and after $T_{0}$, henceforth designated as $T_{-24}$ and $T_{+24}$ respectively. While these times are not strictly storm relative (e.g., Sanders et al. 2013), they identify the antecedent synoptic environment $\left(T_{-24}\right)$ and the persistence of favorable conditions/storm progression $\left(T_{+24}\right)$. Ultimately, five composite patterns each for ice and snow were retained for investigation. These composites were selected and ranked, based on 1) the frequency of occurrence of constituent events (number forming each composite), 2) the rank of the PRPC score by variance explained, 3) the magnitudes of average PRPC loading correlations of constituent events, and 4) the largest numbers of nonduplicative (across PRPCs) events. The last criterion was necessary since some events exceeded the above threshold for more than one PRPC score, indicating weak separation between height fields. For ice, every member of pattern 8 was duplicated in pattern 1 and/or 5, and so was excluded from further consideration. Finally, to provide a thermodynamically holistic characterization of winter storm evolution, the same ice and snow event groups were used to construct associated composites of fields of temperature $(850 \mathrm{hPa}$ and surface $)$, precipitable water, horizontal wind $(250,850$, and $975 \mathrm{hPa})$, and sea level pressure, for times $T_{0}, T_{-24}$, and $T_{+24}$. The resulting synoptic patterns presented below (section $3 \mathrm{~b}$ ) utilize $81 \%$ and $71 \%$ of all events in the ice and snow datasets, respectively.

In addition to aforementioned caveats, further limitations include the following considerations. 1) Small sample size: ideally, the dataset should be expanded for more robust conclusions regarding the representativeness of the derived patterns. At present, the manually intensive nature of the work makes obtaining a larger sample prohibitive. The pattern types shown here, while physically realistic, are liable to change in magnitude and prevalence with an expanded sample, and so this work should be considered as preliminary. 2) Each event had a different domain distribution of ice and/or snow. This study necessitated consideration of a domain rather than a point, with the disadvantage that pattern types are less able to identify expected meteorological conditions at a specific location. A larger sample size allows for individual station synoptic classifications and point statistics. 3) It was noted that, in some cases, height fields visually inconsistent in the amplitude/location of a trough, were placed in the same groups. Variability between events is expected; however, the lower correlation threshold applied in grouping may have aided this disparity. The geopotential height structure of the PRPC-derived scores, and the individual member events in each composite, are 
(a)

(b)

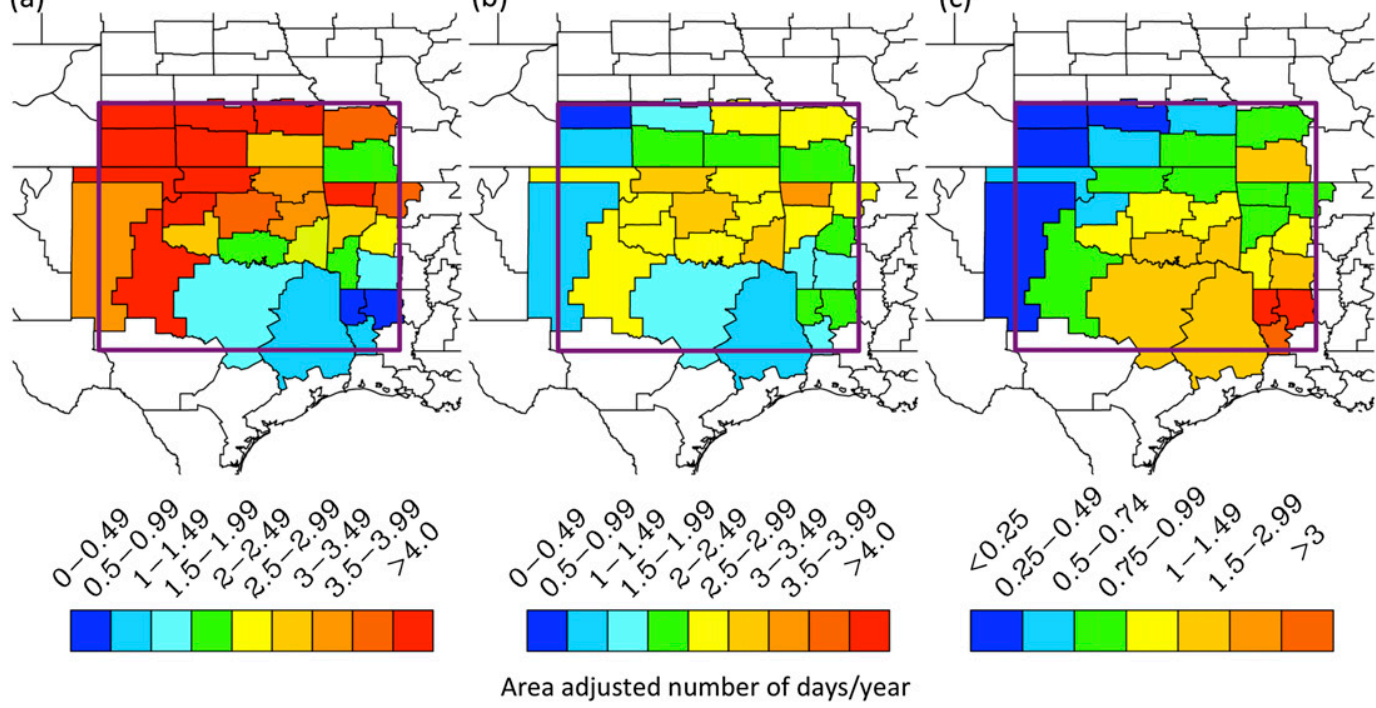

FIG. 2. Spatial distribution of ice and snow events, expressed as the average number of days per year, normalized by the area of each CD to account for their differing sizes. (a) Snow days per year, (b) freezing precipitation (ice) days per year, (c) and ratio of freezing precipitation to snow. Study region is enclosed in the purple box.

provided in the online supplement (see Figs. S1-S5 in the supplement for ice and Figs. S6-S10 for snow). While there is variability, the composite events are generally similar to the PRPC-derived height, and the member events, particularly for the first few patterns in each category. 4) NARR data may not always reflect in situ conditions. A comparison was made of the NARR surface and lower-tropospheric temperature, winds, and moisture for two case studies (one ice, one snow; not shown) with ACRF sounding profiles and Oklahoma Mesonet surface observations (Brock et al. 1995; McPherson et al. 2007). NARR vertical profiles agreed well with the observed results, but peak magnitudes often were underestimated. For the case studies considered, NARR near-surface temperatures were almost always warmer than the observations, particularly near noon central standard time (CST). Spatial comparisons with interpolated mesonet data revealed a slower southward progression and a more zonal orientation of the $0^{\circ} \mathrm{C}$ isotherm, particularly for the ice case study. NARR may therefore incorrectly represent the intensity of surface cold air masses under certain conditions, possibly resulting from inadequate treatment by the Eta Model's planetary boundary layer and radiation schemes (e.g., Kennedy et al.2011). As a result of this likely surface warm bias, all composite patterns (Figs. 6-15) used the $+1^{\circ} \mathrm{C}$ surface isotherm instead of $0^{\circ} \mathrm{C}$ to represent the freezing line, hereafter designated the "freezing line" or "freezing isotherm."

\section{Results}

\section{a. Spatial climatology and thermal profile}

The distribution of ice events over the SGP (Fig. 2b) revealed a freezing precipitation maximum in the central and northeastern domain, including a southwestnortheast-orientated region of higher ice frequency also noted by Grout et al. (2012). In contrast, snow is more frequent in the west and north (Fig. 2a), and regions of elevated terrain in the east (Ozark mountains of northwest Arkansas). The higher-altitude western subregion ( $\sim 1-\mathrm{km}$ increase from east to west; Fig. 1) promotes this increased snowfall. This region also is farther from the low-level warm moist air necessary for freezing rain. These results agree with a study of the spatial distribution of snow events in Oklahoma exceeding $4 \mathrm{in}$. (Branick 2001), by showing a general northwest-southeast gradient in snow frequency. The ratio of freezing precipitation to snow events has a northwest-to-southeast increase, reflecting the changes in both parameters (Fig. 2c). The somewhat discontinuous character of Fig. 2 may reflect variable $\mathrm{CD}$ shape and size, possible discrepancies in reporting frequencies (e.g., Branick 1997), and precipitation modulation by local topography.

Figure 3 shows the average winter (November-March) monthly distributions of freezing precipitation and snow by study region quadrant. Freezing precipitation events primarily occurred in December-February, with peak frequencies in December and January (Changnon and Karl 2003). This seasonality is consistent with freezing 
(a)

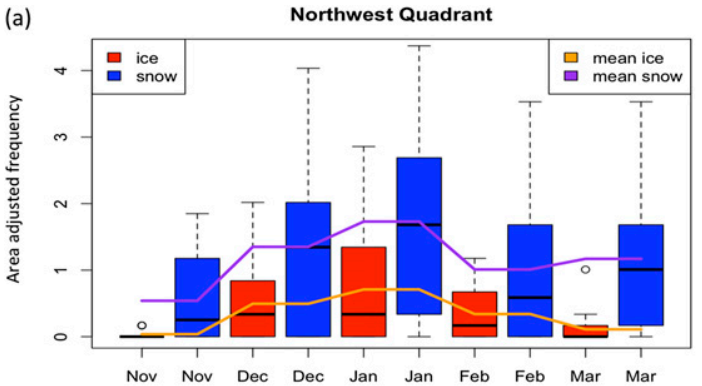

(c)

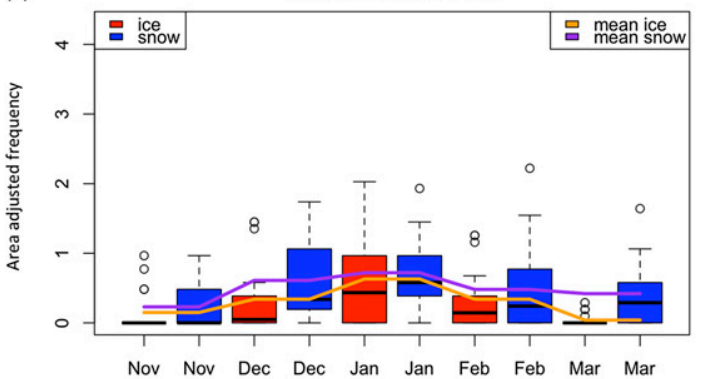

(b)

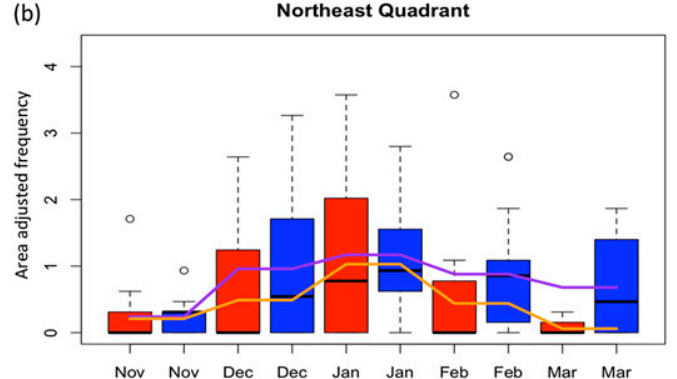

(d)

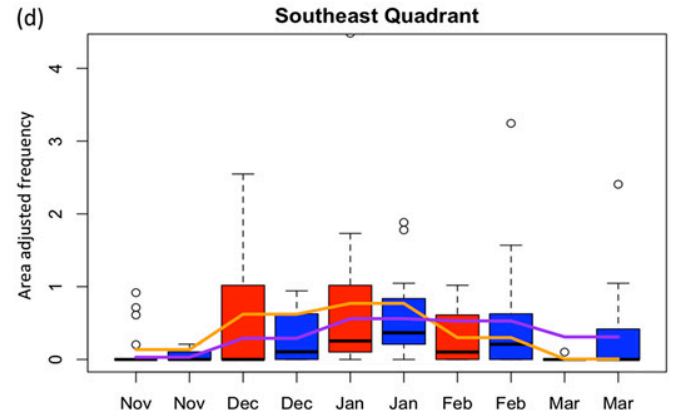

FIG. 3. Average monthly variation of ice and snow events by quadrant during November-March. Frequency is number of days per season, normalized (weighted) by area of each $\mathrm{CD}$, with each $\mathrm{CD}$ assigned quadrants in which $\geq 50 \%$ of the $\mathrm{CD}$ area resides. Box-and-whisker diagram showing the median (thick black line), interquartile range (colored box), 10th and 90th percentiles (horizontal bar), and extreme values ( $>90 \%$ and $<10 \%$ ) (circles) for each month, valid 1993-2011. Lines give the monthly average normalized frequency for ice and snow.

precipitation requiring a shallow layer of subfreezing air, which Rauber et al. (2001) suggest frequently occurs within and along the leading edge of a shallow cold anticyclone, which would typically develop over snowcovered high latitudes. These air masses migrate south into the SGP primarily from the Canadian Arctic, where snow cover is of maximum depth and extent during midwinter (e.g., Wang et al. 1995). Snow events in the northern study domain exhibit a broader seasonal distribution, including a peak during December-January, a slight decrease during February, and a second peak in March (Figs. 3a,b). Both precipitation types are generally infrequent (typically $<4$ days $\mathrm{yr}^{-1}$ ), particularly in the southern subdomains.

Figure 4 summarizes the vertical thermal profiles of each precipitation type, using environmental melting and freezing potentials, which measure the potential for melting and freezing of hydrometeors, based on differences between ambient wet-bulb temperature and $0^{\circ} \mathrm{C}$, integrated over a layer. The mathematical formulation is given by Robbins and Cortinas (2002) and Mullens et al. (2016). Freezing rain (57 cases) is split into ice storm and "glaze" subsets, where the former are events where local ice accumulations exceeded $0.25 \mathrm{in}$. over the storm duration. The ice storm thermal profile ( 25 cases) is distinct in the depth and magnitude of its warm layer, while the refreezing layer is weaker. Based on bootstrapping of the mean using 250 replications (not shown), ice storm wet-bulb temperatures were statistically distinct ( $95 \%$ confidence interval). This result should be interpreted with some caution, however, as the sample was weighted toward long-duration and highly impactful ice events, over which multiple sounding profiles (at different times and locations), were obtained. Light freezing rain and freezing drizzle evidenced cooler and/or shallower warm layers and more pronounced refreezing layers, while the refreezing-layer magnitude was most pronounced for ice pellets, as anticipated, based on prior observations and the body of literature (e.g., Hanesiak and Stewart 1995; Stewart and King 1987; Zerr 1997).

Figure 5 highlights surface and 850 -hPa wind profile characteristics, by precipitation type, including freezing rain, but excluding its further partitioning to glaze and ice storm. Common freezing rain and drizzle surface winds have a northerly and easterly component (Figs. 5a,b), whereas northerly winds are more prevalent for ice pellets and snow (Figs. 5c,d). Wind speeds in the snow sample indicated higher peak values than the other precipitation types; however, bootstrapped confidence intervals from 250 replications around the means at both levels (not shown) did not reveal statistically distinct 


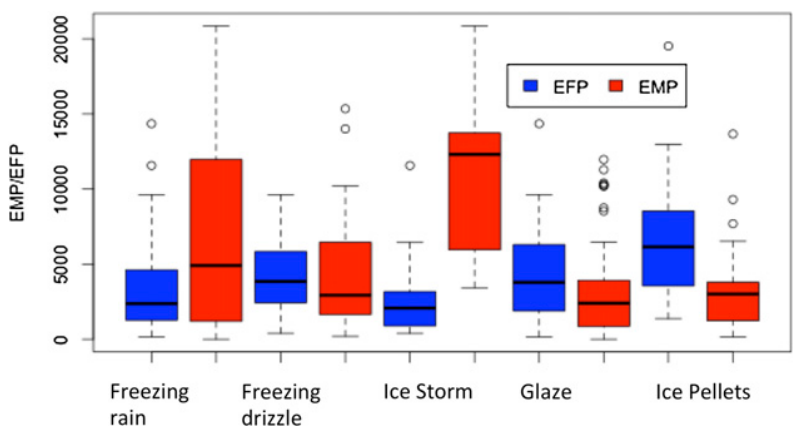

FIG. 4. Boxplot distributions of environmental melting and freezing potentials (units of ${ }^{\circ} \mathrm{Cm}$ ) for freezing precipitation, including all freezing rain $(n=57)$, freezing drizzle $(n=21)$, and ice pellets $(n=19)$. Freezing rain is also divided into ice storm $(n=25)$ and "light" categories $(n=32)$, based on ice accumulation in the vicinity of a sounding station site.

differences in wind speed. Aloft, freezing rain and freezing drizzle winds were predominantly southerly and southwesterly, backing toward the south and southeast and more variable for ice pellets. Approximately $60 \%(20 \%)$ of the 850 -hPa winds sampled during snowfall had a northerly (southerly) component (Fig. 5d).

\section{b. Synoptic patterns: Composite analysis and description}

The five leading mid- to upper-tropospheric composite circulation patterns for ice- and snow-dominated events, using the approach described in section $2 b$, are displayed in panel a of Figs. 6-15. The order of the patterns (1-5) was also described in section 2b. Each composite evolution depicts one possible scenario concerning the expected duration, magnitude, and approximate location for the indicated phase type. Locations of event-specific ice or snow by pattern are displayed at the top of each figure. These were estimated from the daily average NARR categorical precipitation type $\geq 10 \%(0.1)$. In addition to the PRPC-derived midtropospheric circulation, Figs. 6-15 present corresponding composites of sea level pressure (panel $\mathrm{b}$ in each figure), 850-hPa standardized temperature anomalies relative to a 1979-2012 mean and standard deviation (hereafter $\sigma$; panel $\mathrm{c}$ in each figure), and precipitable water vapor (PWV; panel d in each figure). Three categories are used below to assist in the explanation and comparison of key features, based on visual inspection of the location and amplitude of the 500-hPa trough axis at $T_{0}$. All variables are at $1^{\circ} \times 1^{\circ}$ horizontal resolution.

\section{1) High-Amplitude trough PROXimal TO THE DOMAIN}

Patterns within this definition include ice patterns 1 and 5 and snow patterns 2,4 , and 5. Ice pattern 1 (Fig. 6 ) depicts a positively tilted trough over the southwestern United States at $T_{-24}$, bounded to the northeast by westnorthwesterly flow (Fig. 6a). By $T_{0}$, the trough axis is located just west of the domain, with diffluent flow and ascent promoted via divergence associated with the leftexit/right-entrance regions of a split jet streak circulation. SLP (Fig. 6b) at $T_{-24}$ shows high pressure over the central and eastern United States, corresponding to southeastward advection of a cool air mass (Fig. 6c). By $T_{0}, 850-\mathrm{hPa}$ temperatures modestly increase in the southeast $(\sim+0.5 \sigma)$, while cold air builds into the southern high plains (Fig. 6c). Strengthening low-level flow from the western Gulf of Mexico (GOM) advects moisture (Figs. 6b,d), with PWV departures maximizing at $+1.5 \sigma$ in the central and south domains (Fig. 6d). At $T_{0}$, the composite average thermal zone favorable for ice (demarked by the area where the $850-\mathrm{hPa}$ freezing isotherm is north of its surface counterpart) encompasses the western through northeastern sections of the domain. However, there is variability in the spatial distribution of the freezing rain and ice pellets by event, with some clustering in the east (Fig. 6, top). Precise locations of ice are a function of the southward extent of subzero surface temperatures, the position of the trough axis with respect to the region, and the development/movement of a surface low pressure. By $T_{+24}$, the composite midlevel low resides over the domain. This cold-air advection (CAA) aloft can increase snowfall potential, if sufficient moisture remains in place.

Ice pattern 1 compares visually with composite snow pattern 2 (Fig. 7). However, in the latter case, the ridge is further amplified over the eastern United States (Fig. 7a), corresponding to a warmer low-level air mass (Fig. 7c; $T_{-24}, T_{0}$ ) not present for ice pattern 1 (likely resulting from the cooler surface air mass acting to reduce the column thickness). The absence of surface cold air contributed by an Arctic high likely precludes development of a pronounced inversion layer aloft and, thus, sustained freezing precipitation. By $T_{0}$, a ridge amplifies east of the trough, associated with warm-air advection (WAA) and temperature increases (e.g., Ressler et al. 2012), while moisture advection builds positive PWV anomalies (Fig. 7d). A deeper composite surface low pressure center is evident through the 48 -h period, situated near the Arklatex region (including parts of Arkansas-Louisiana-Texas) by $T_{+24}$ (Fig. 7b), with movement northeastward from the lee of the mountainous terrain in northern Mexico. The faster eastward movement of the trough through its evolution is facilitated by a strong jet streak southeast of its axis. The location of accumulating snow (Fig. 7, top) relates to the specific track of the low and its attendant moisture and temperature structure. Commonly impacted locations 
(a)

FZRA
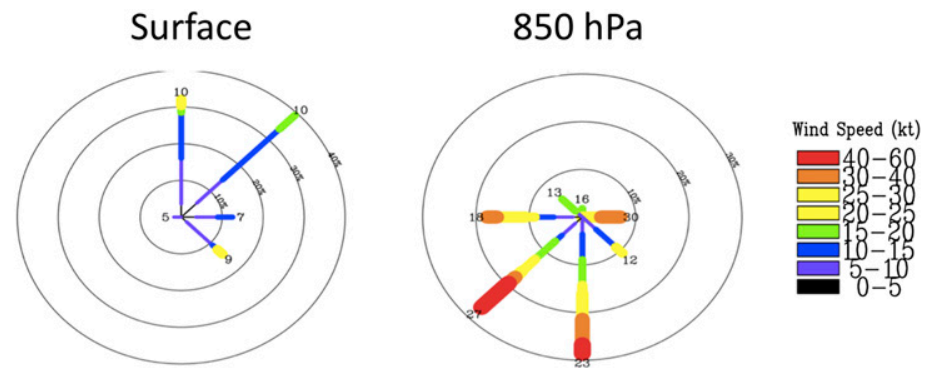

(b)

FZDR
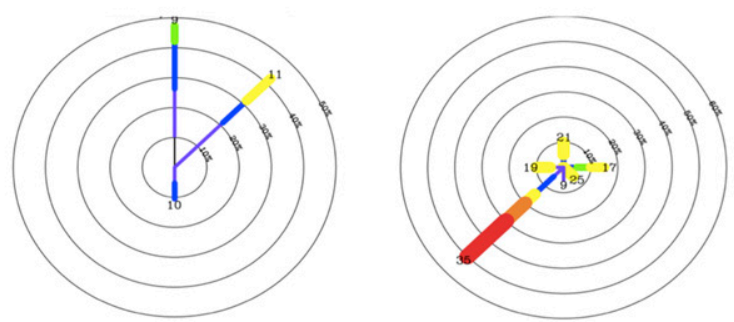

(c)
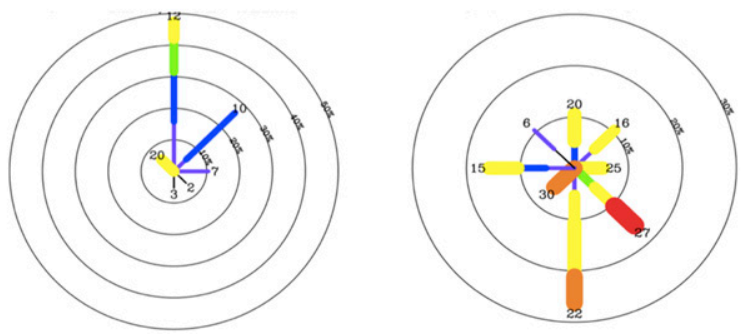

(d)
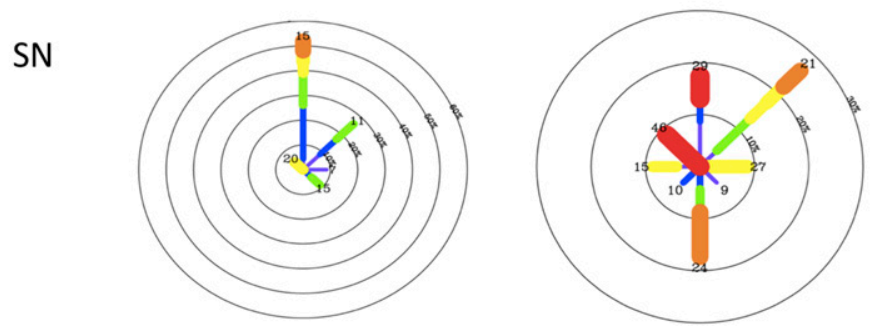

FIG. 5. Wind roses for (a) freezing rain, (b) freezing drizzle, (c) ice pellets, and (d) snow at the (left) surface and (right) 850-hPa layer. Directions follow standard compass format. Concentric rings indicate frequency increments of $10 \%$, while shading is wind speed (kt). Numbers shown are mean wind speed. Values are binned into eight directional bounds, N, NE, E, etc.

include the northern and western domains northnorthwest of the low pressure center.

The evolution of snow pattern 4 (Fig. 8) is similar to snow pattern 2, albeit the composite jet upwind of the trough axis is stronger, with the associated input of cyclonic vorticity to the trough promoting digging and amplification into the domain at $T_{0}$ (Fig. 8a). The central pressure of this composite surface cyclone is the lowest of all the patterns (Fig. 8b). While moisture and temperature advection east of the trough do not reveal distinct anomalous positive departures $(\leq+1 \sigma)$, the very low temperatures/PWVs west of the low are notable (Figs. 8c,d). Both snow patterns 2 and 4 can be associated with heavy snowfall, with snow pattern 4 evidencing stronger northerly surface winds (Fig. 8b). Snow accumulation associated with events in this composite (Fig. 8, top) is typically over the north and eastern domain.

The composite trough in ice pattern 5 (Fig. 9) moves eastward from Baja California at $T_{-24}$ to Arizona/New Mexico at $T_{0}$ (Fig. 9a). The domain is located under the right-entrance region of a jet streak over the northeast United States, associated with an exiting low. A cool air mass is already positioned through the central and southern United States at $T_{-24}$. This cool air mass, and composite anticyclone, are strengthened by $T_{0}$ (Figs. $9 \mathrm{~b}, \mathrm{c}$ ), but to the south, WAA strengthens the across-domain 


\section{Composite Ice Pattern 1 $(\mathbf{n}=\mathbf{8})$}

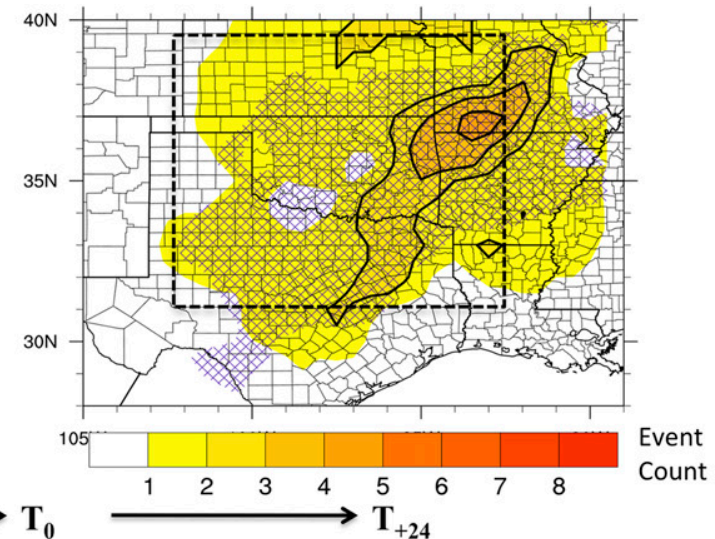

(a)
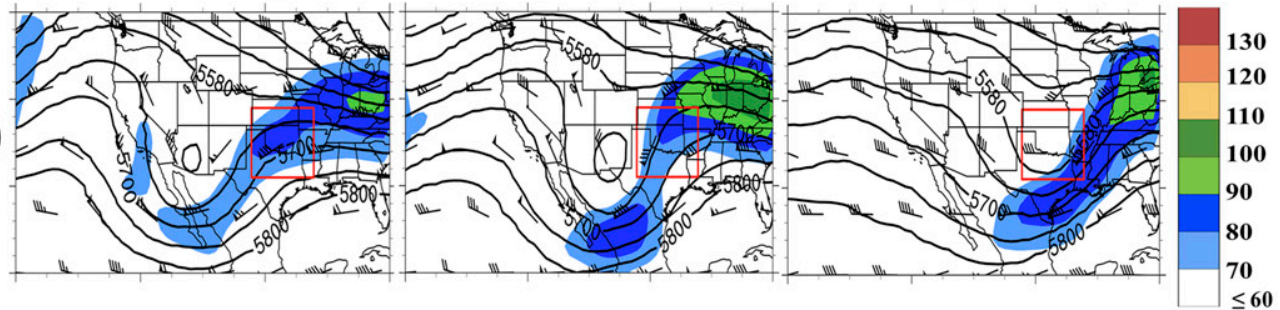

(b)
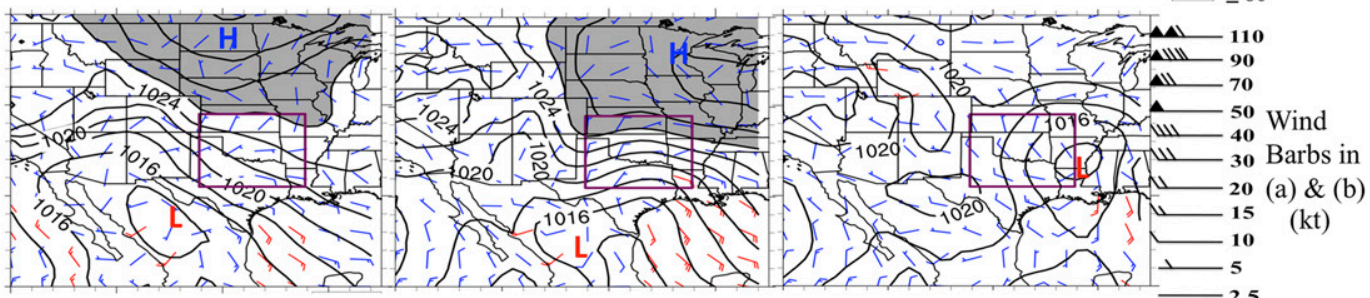

(c)
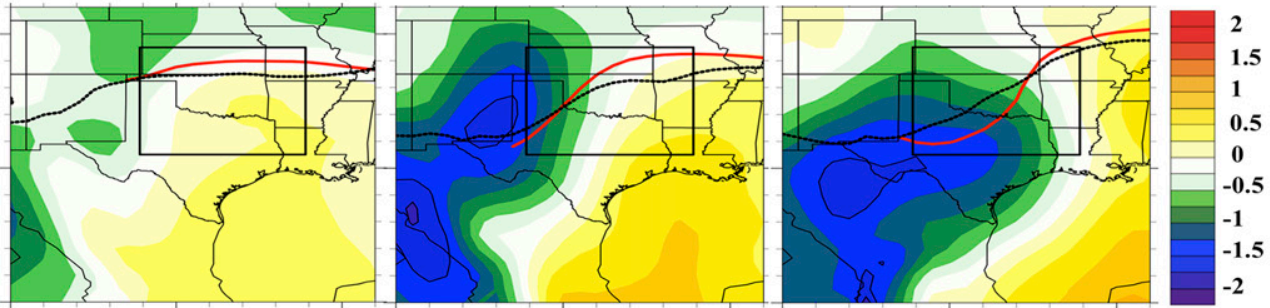

\section{$\mathrm{T}$}

$(\sigma, \mathrm{K})$
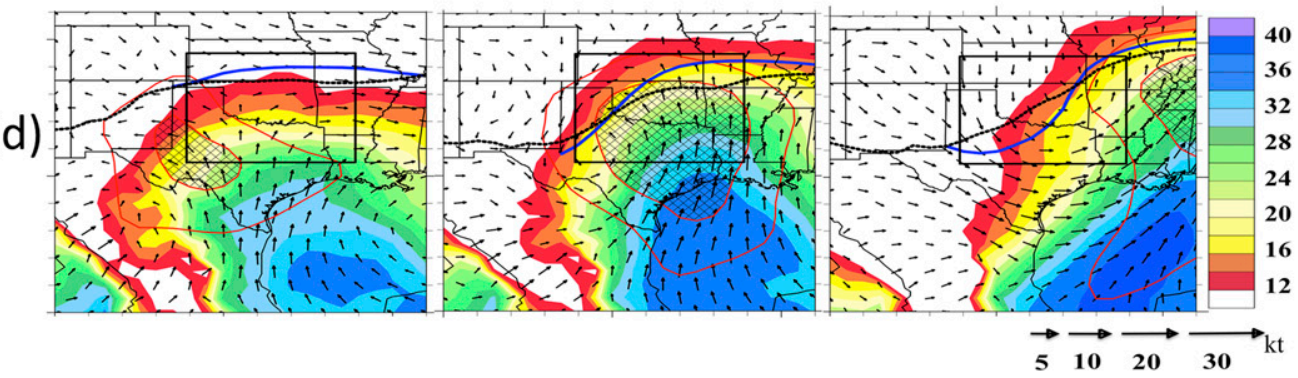

PWV

$(\mathrm{mm})$

FIG. 6. Multipanel plot displaying evolution of composite ice pattern 1 at (left) $T_{-24}$, (center) $T_{0}$, and (right) $T_{+24}$. Top figure displays the locations of freezing rain (shaded) and ice pellets (stippled) for each constituent event forming the composite, and the number of events impacting each grid point for freezing rain is summed. Data are NARR reanalysis categorical freezing rain or ice pellets $>0.1$ averaged over 1 day. The four subsequent rows display the (a) $500-\mathrm{hPa}$ geopotential height (m) and 250-hPa winds (kt); (b) SLP (hPa), where dark gray (light gray) is $<1012 \mathrm{hPa}(>1026 \mathrm{hPa})$, and 975-hPa wind barbs (blue is $<15 \mathrm{kt}$; red is $>15 \mathrm{kt}$ ); (c) standardized temperature anomalies, relative to a 1979-2012 mean and standard deviation $\sigma$, expressed as a departure from $\sigma$, with surface $1^{\circ} \mathrm{C}$ and $850-\mathrm{hPa} 0^{\circ} \mathrm{C}$ isotherms overlaid in black and red, respectively (because of the terrain the 850-hPa isotherm ends at $104^{\circ} \mathrm{W}$ ); and (d) PWV, with $850-\mathrm{hPa}$ wind vectors $(\mathrm{kt})$. Red-lined (stippled) regions indicate standardized PWV anomalies exceeding $\pm 1 \sigma(+1.5 \sigma)$, while surface and $850-\mathrm{hPa}$ isotherms are also overlaid as in (c), with $850 \mathrm{hPa}$ in blue. In each figure, the SGP domain is outlined. 


\section{Composite Snow Pattern 2 $(\mathbf{n}=\mathbf{1 0})$}

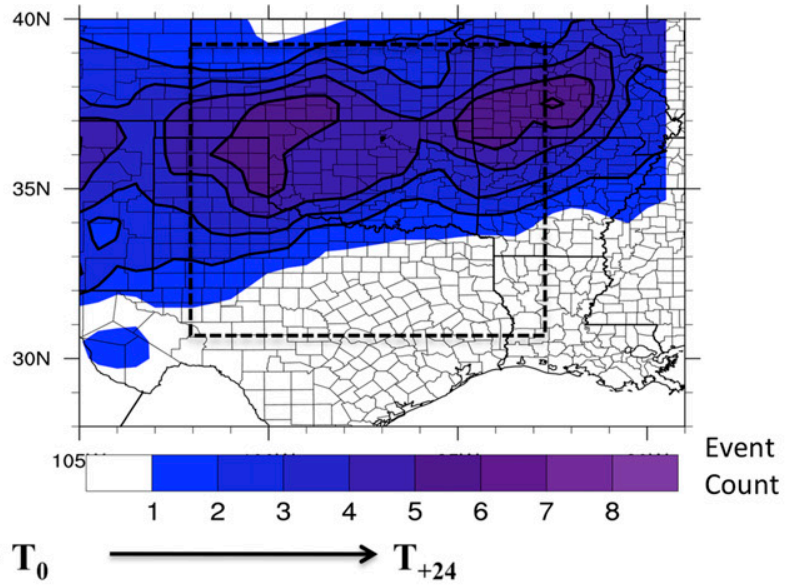

(a)
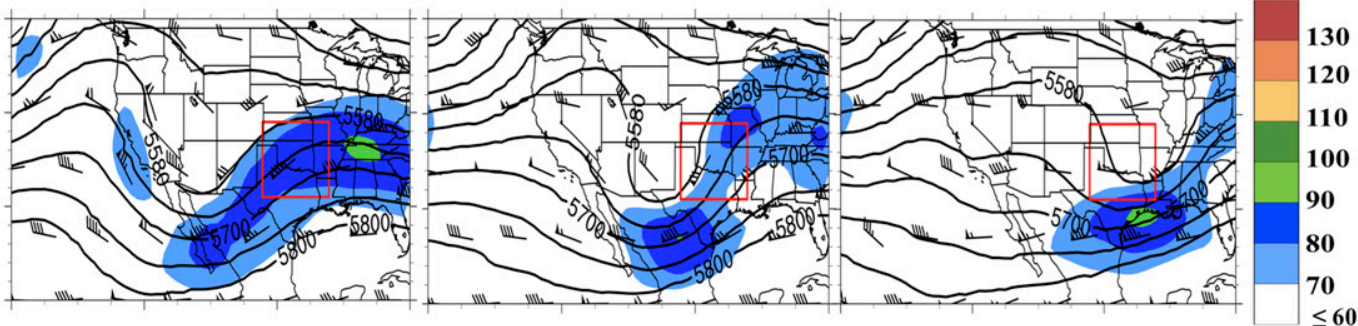

(b)
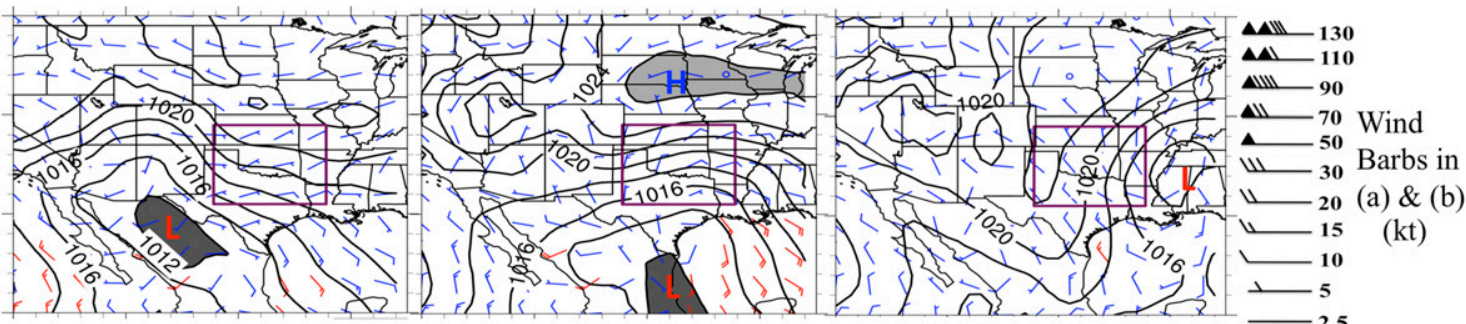

(c)
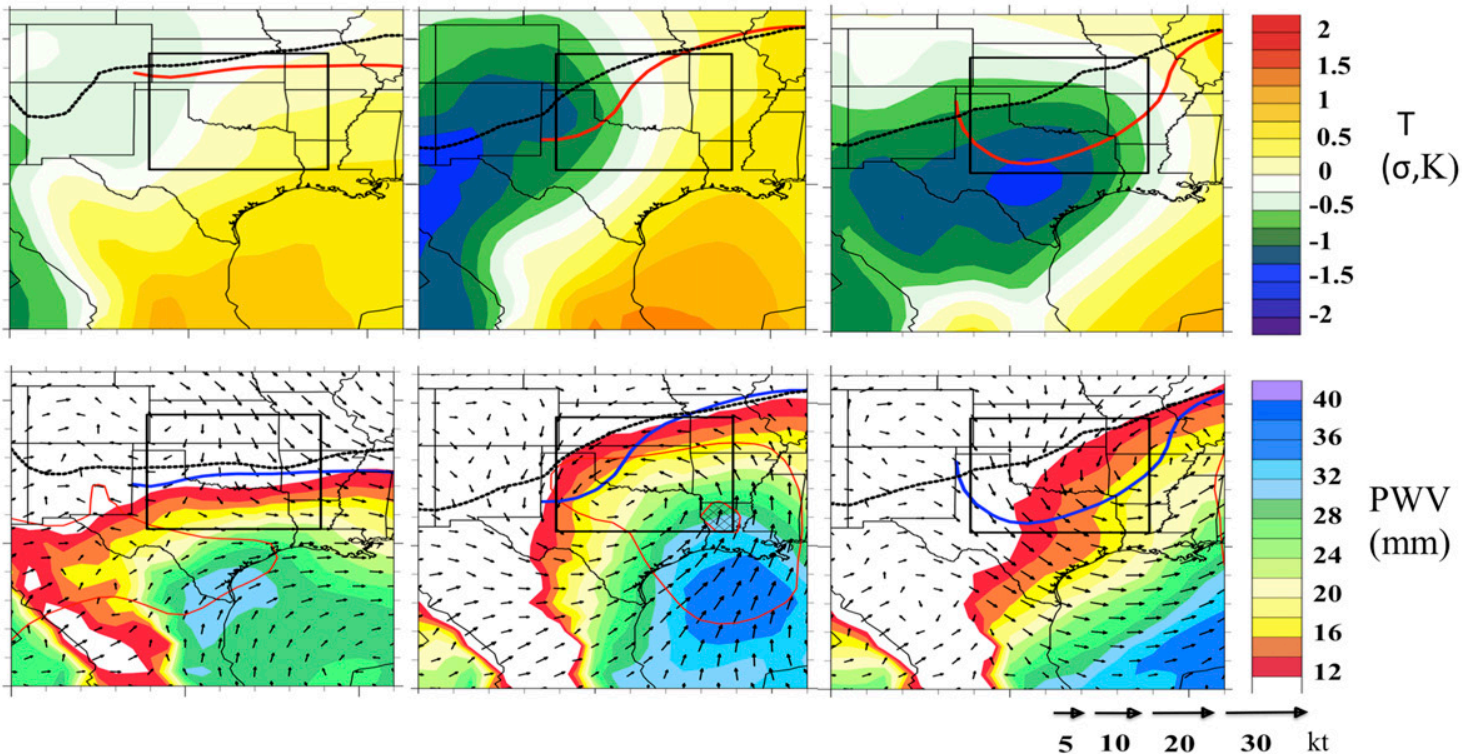

FIG. 7. As in Fig. 6, but for snow pattern 2, with the exception that the top panel displays the locations and number of events by grid point for snow, based on all constituent events forming the composite. 


\section{Composite Snow Pattern 4 $(n=6)$}

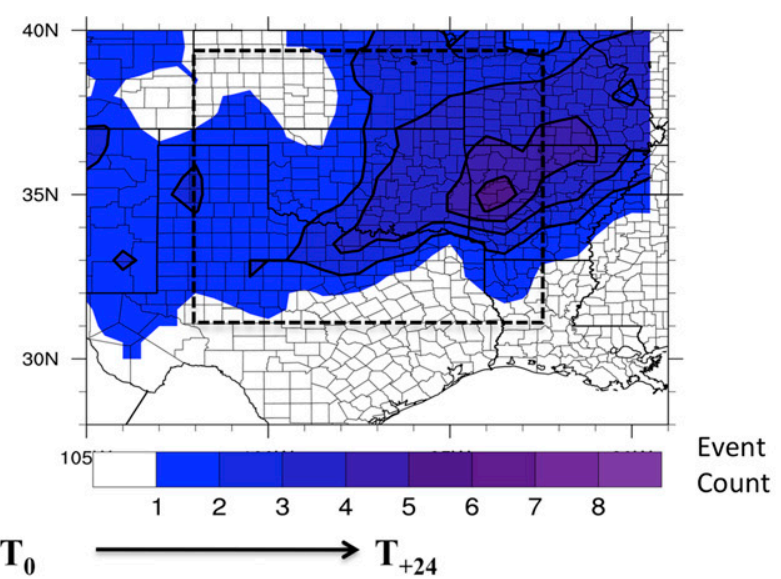

(a)
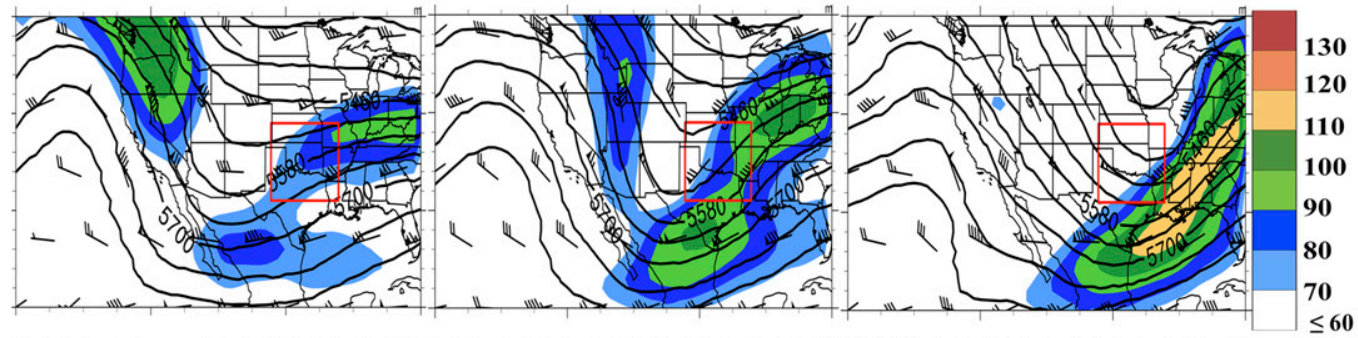

(b)
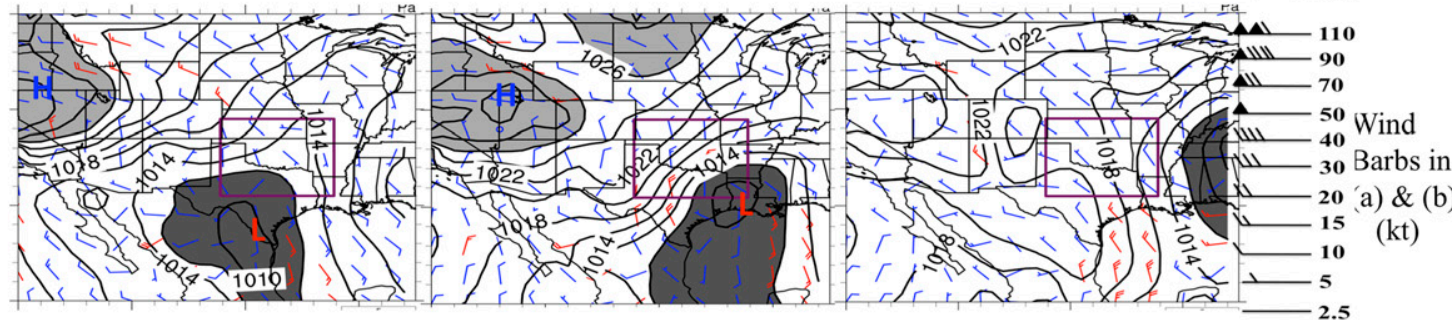

(c)
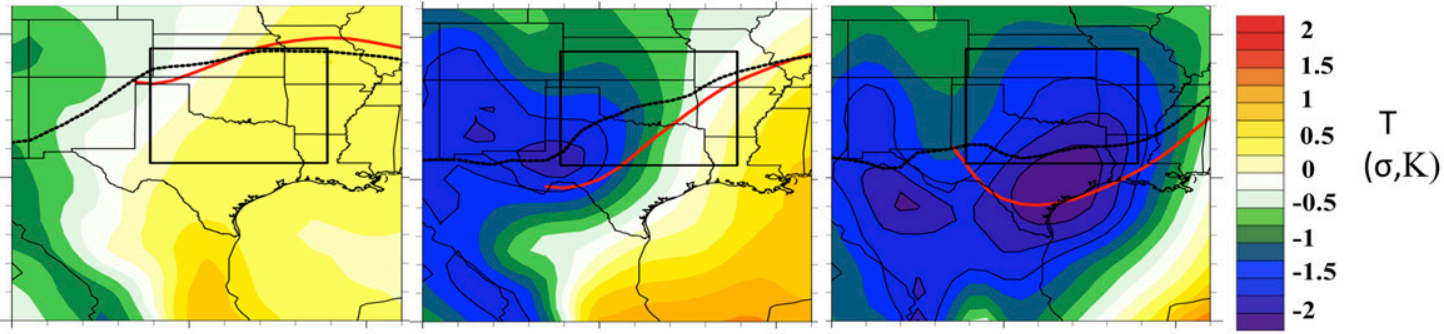

(d)
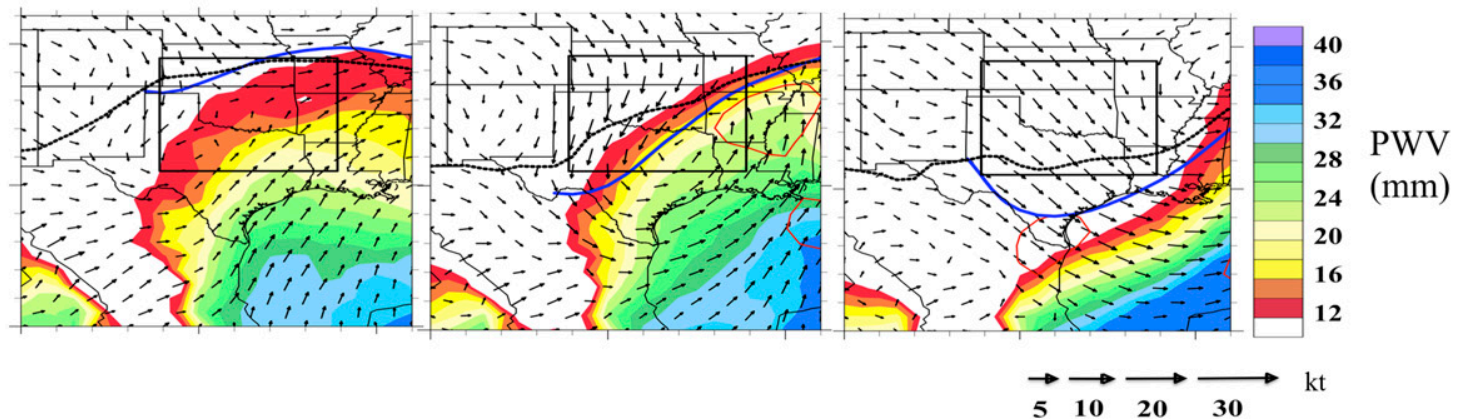

FIG. 8. As in Fig. 7, but for snow pattern 4 


\section{Composite Ice Pattern 5} $(\mathrm{n}=6)$

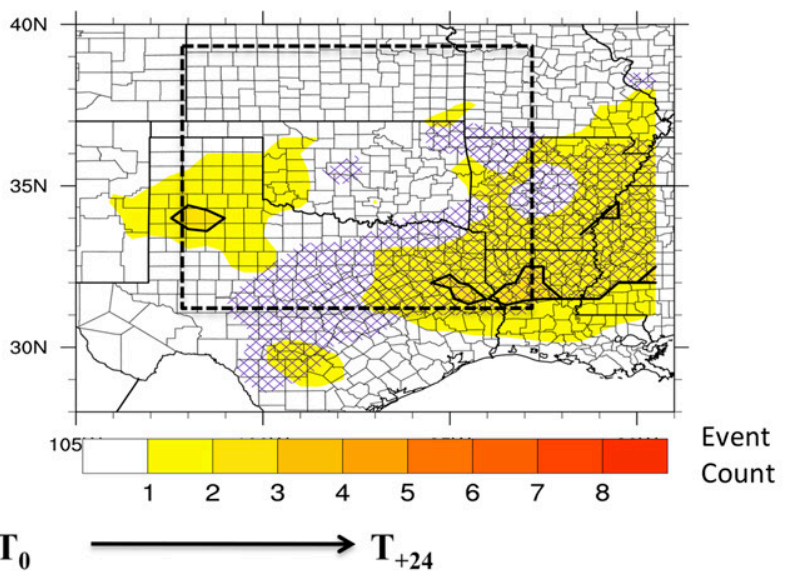<smiles>[124I]C[141I]</smiles>

(a)
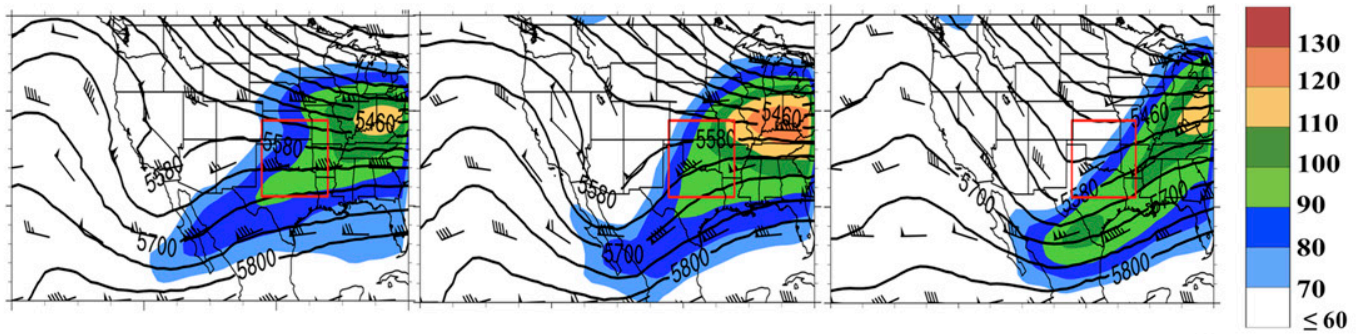

(b)

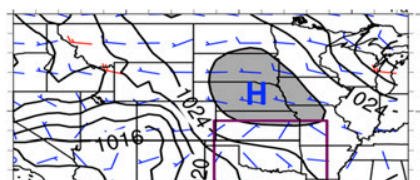

(c)

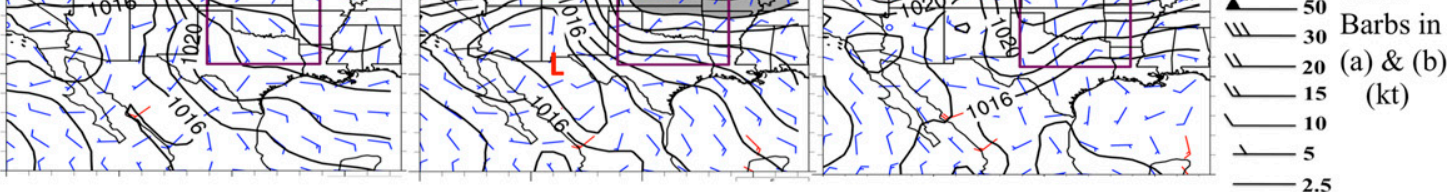

(d)
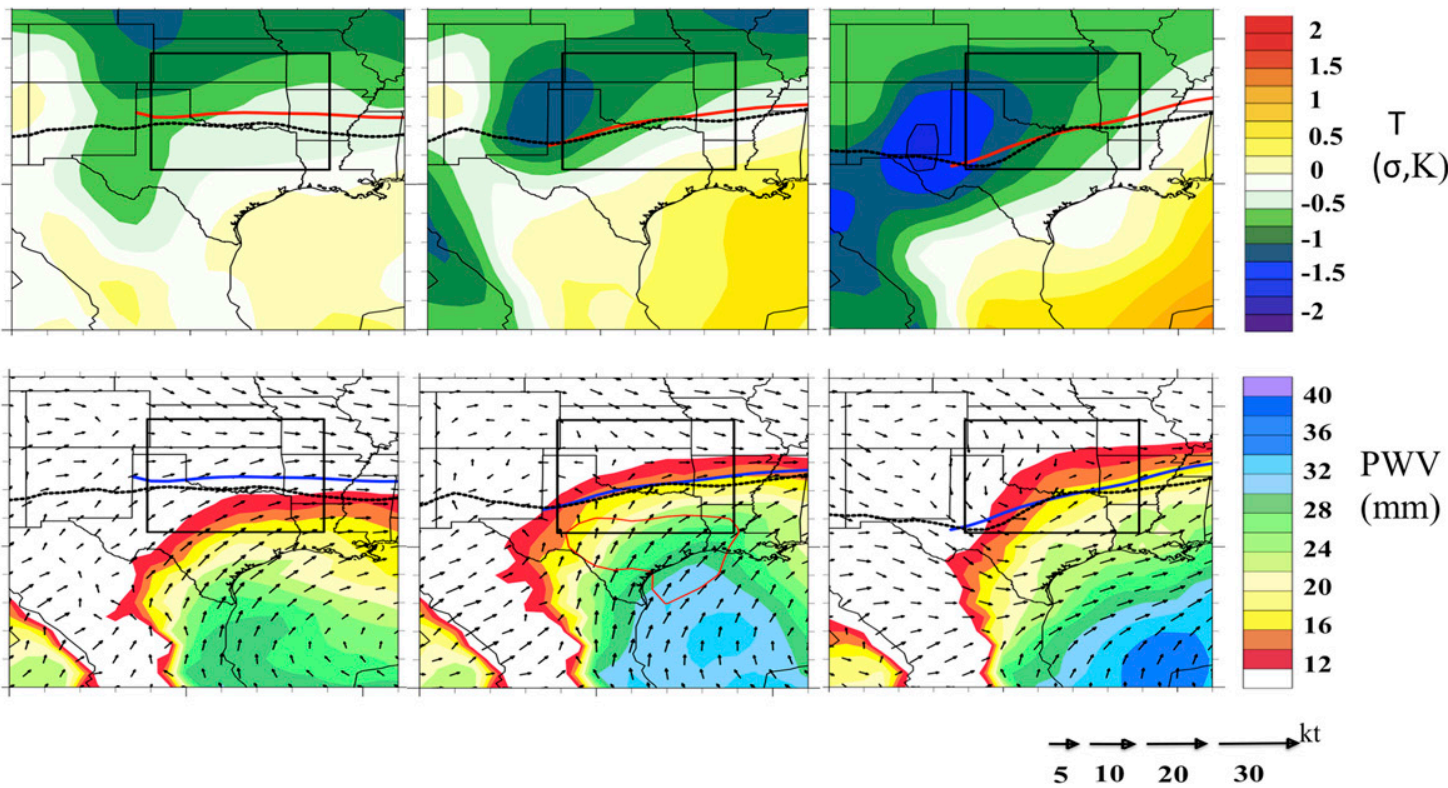

FIG. 9. As in Fig. 6, but for ice pattern 5. 


\section{Composite Snow Pattern 5$$
(\mathbf{n}=6)
$$

(a)
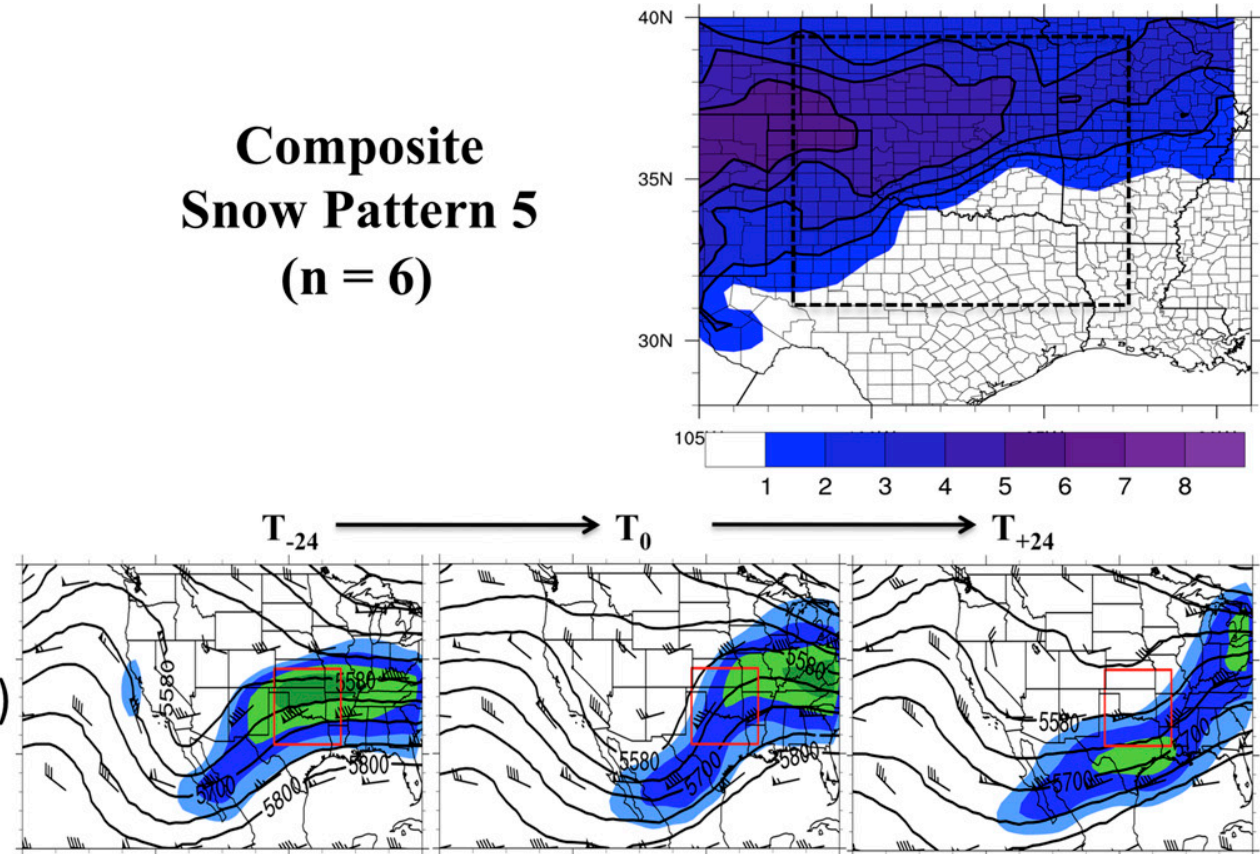

Event

Count

(b)
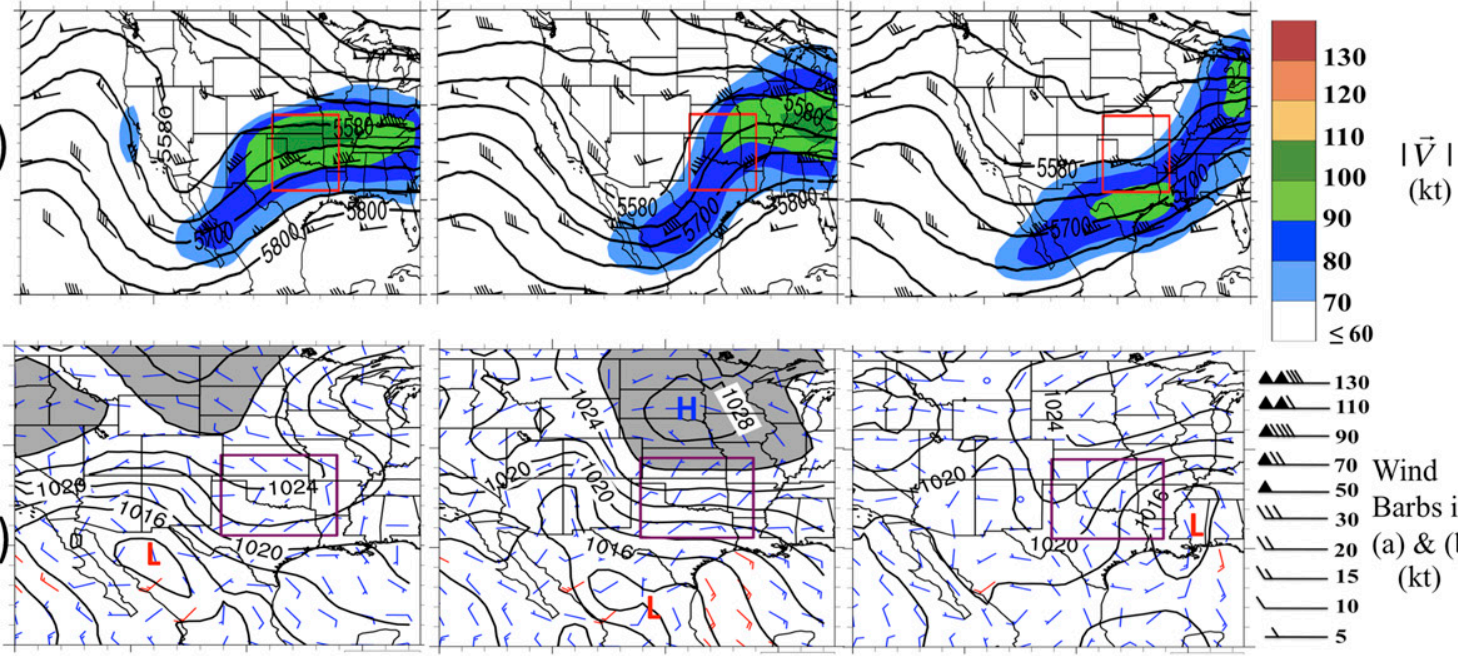

(kt)

(c)
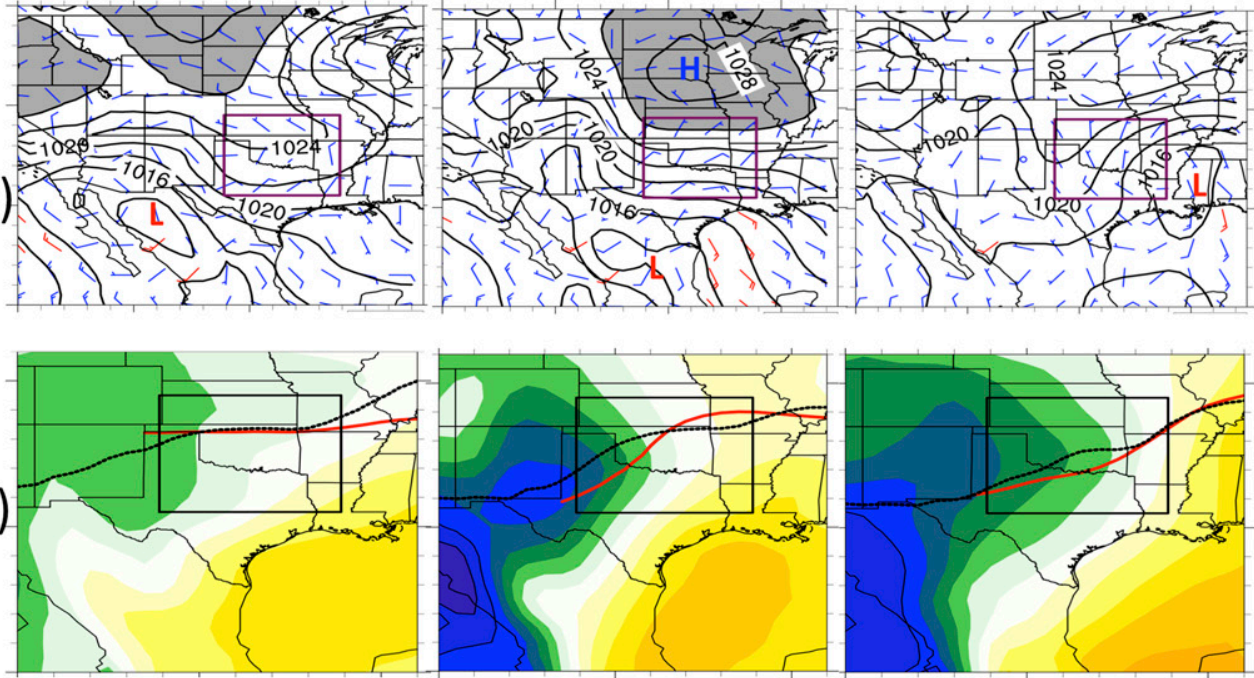

ANIII 130

$\Delta \sqrt{11}-110$

all 90 - 70 Wind

U_ 50 Barbs i

$4 \quad 20$ (a) \& (b)

15 (kt)

(d)
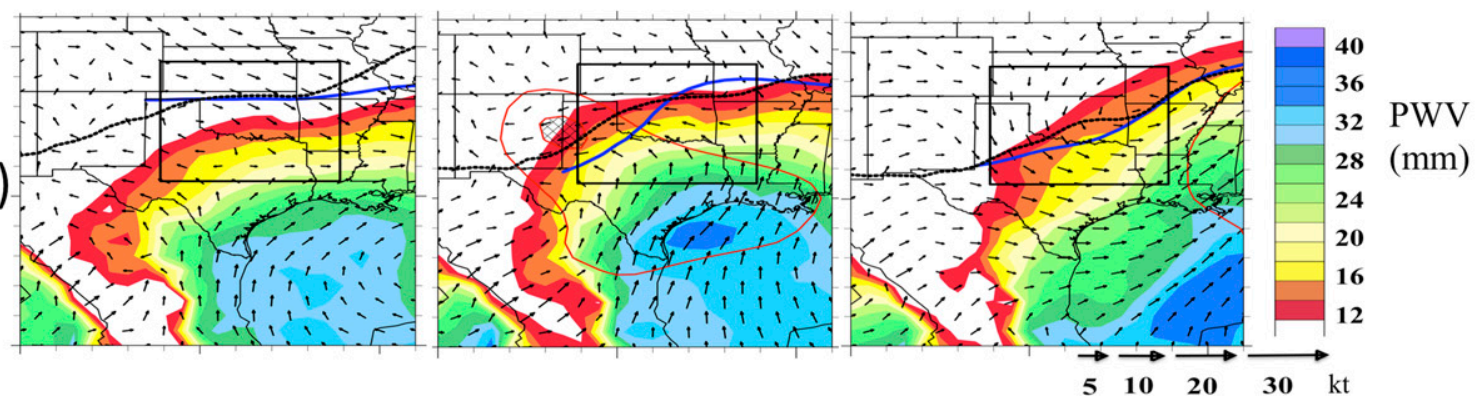

FIG. 10. As Fig. 7, but for snow pattern 5. 


\section{Composite Ice Pattern 4 $(\mathbf{n}=5)$}

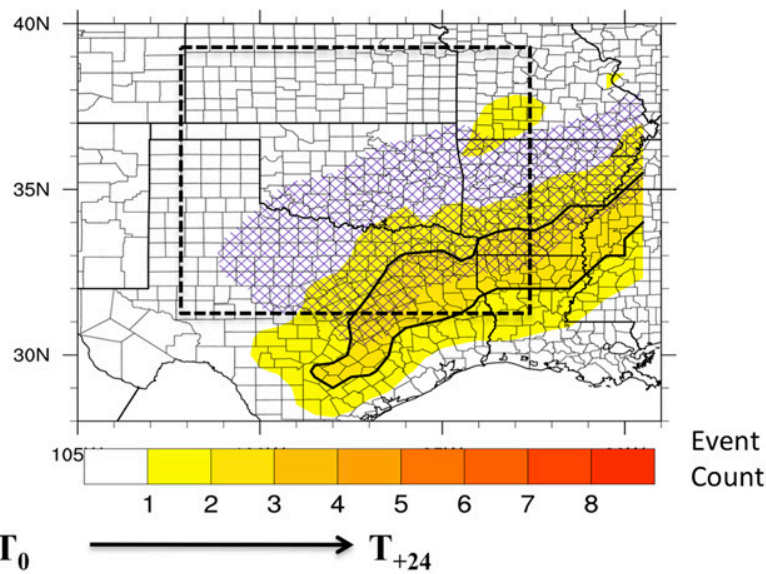

(a)
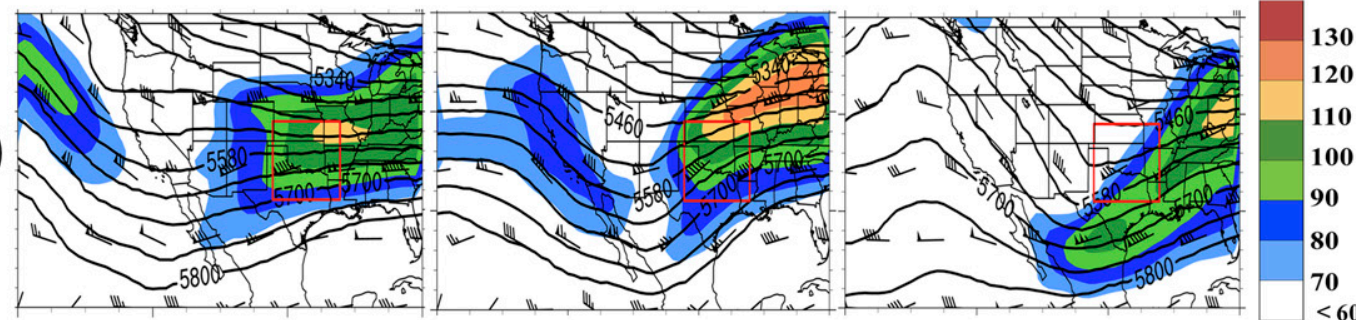

(b)

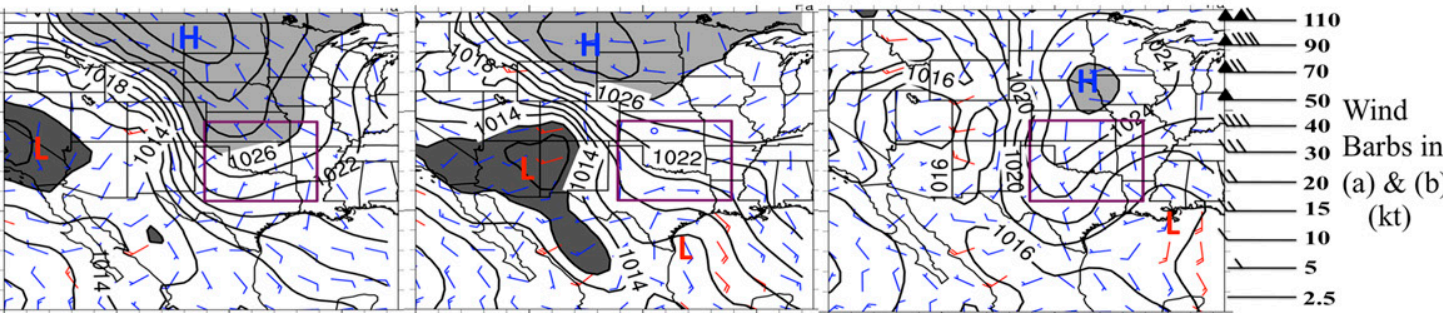

(c)
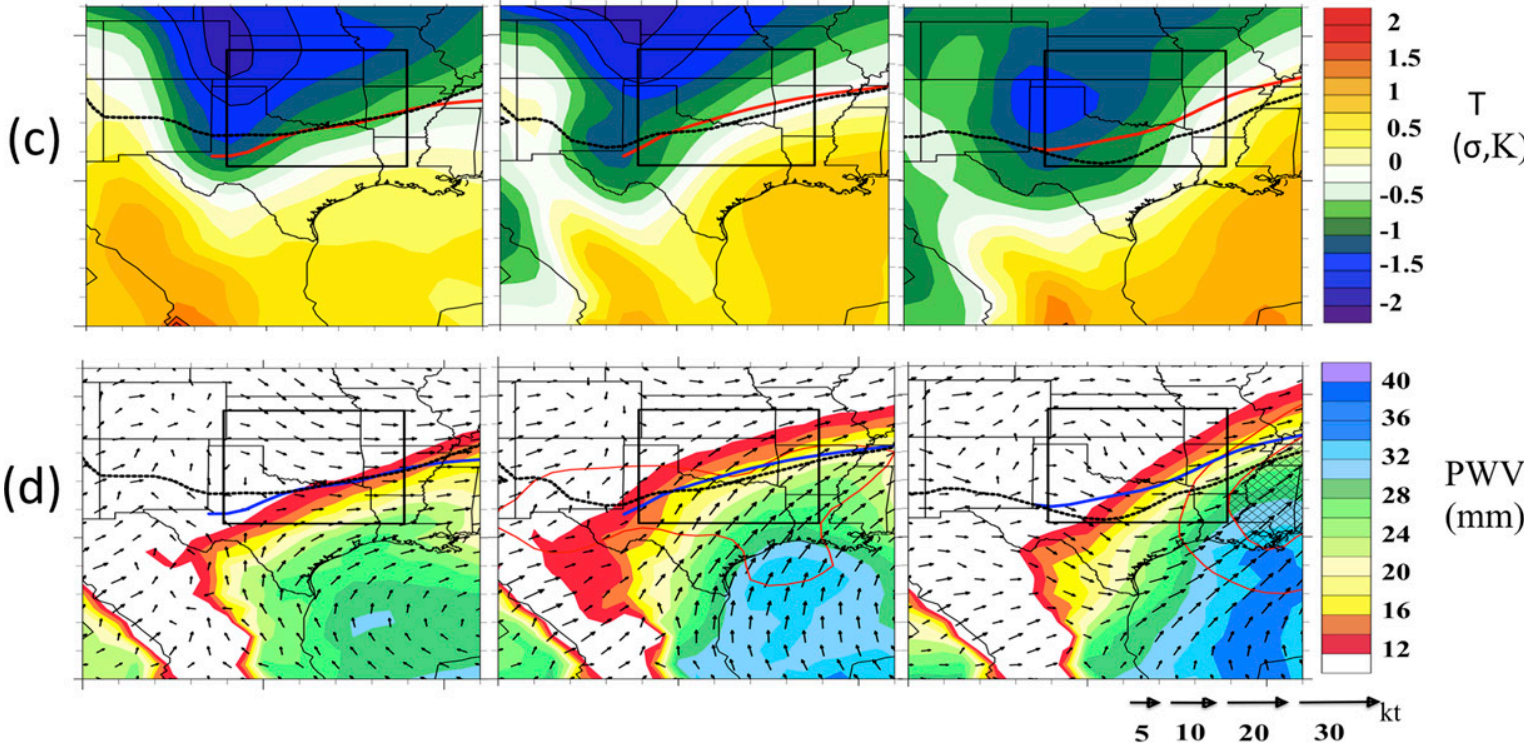

FIG. 11. As in Fig. 6, but for ice pattern 4. 


\section{Composite Snow Pattern 1 \\ $(\mathbf{n}=8)$}

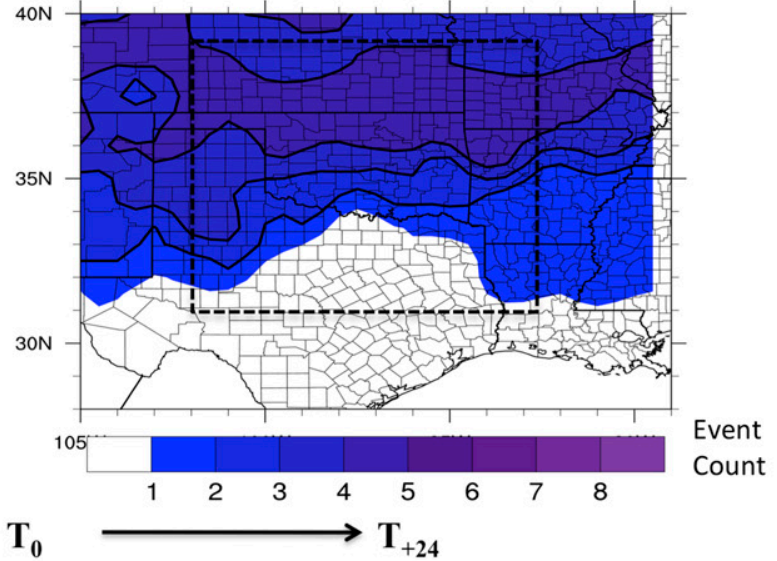

(a)
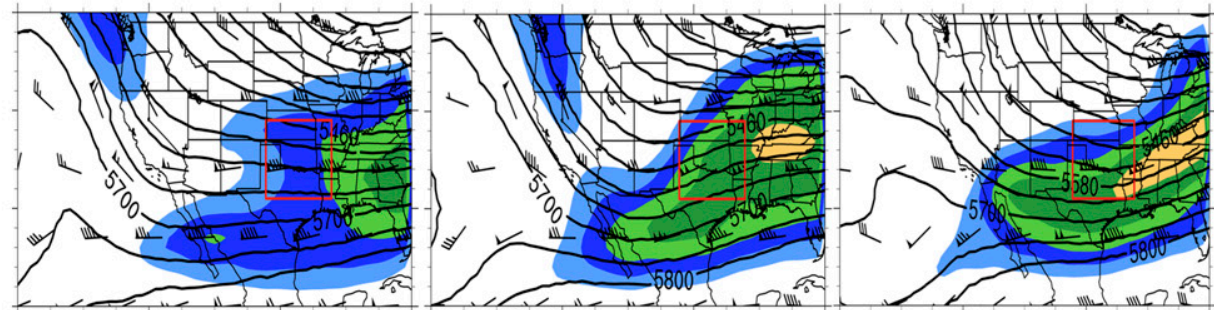

$|\vec{V}|$

(kt)

(b)
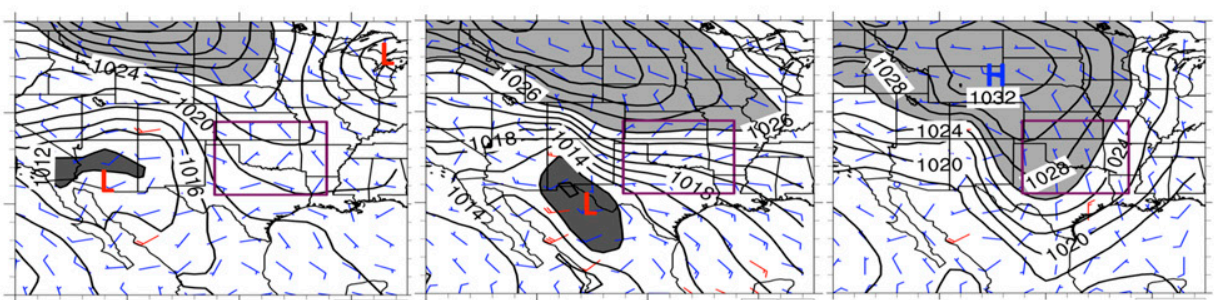

$\begin{array}{lll}\Delta 11 & 110 \\ 4 & 90\end{array}$

$411 \quad 70$

$\Delta-50$
4

w_ 30 Barbs in

U_ 20 (a) \& (b)

Dits
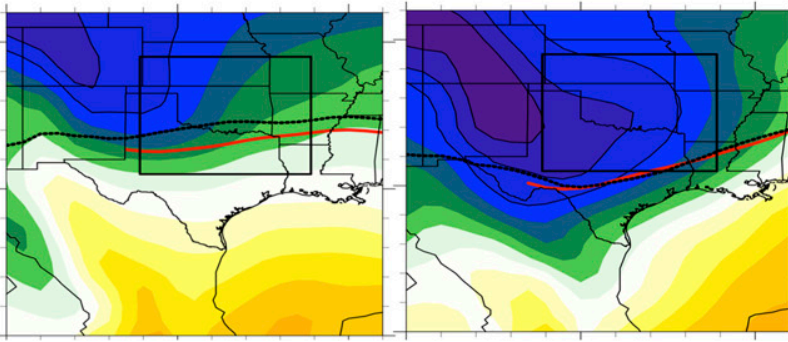

$\lcm{1}$

(c)
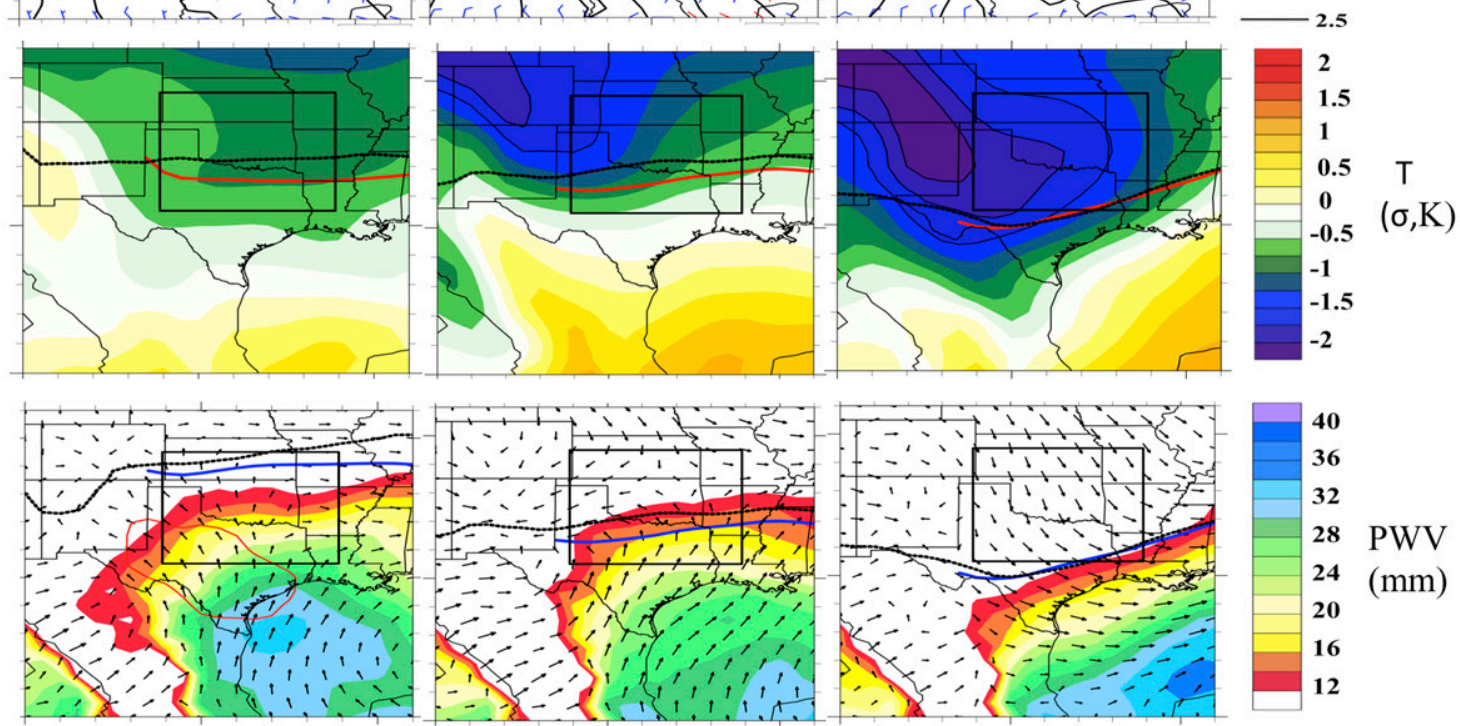

(d)

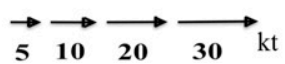

FIG. 12. As in Fig. 7, but for snow pattern 1. 


\section{Composite Snow Pattern 3 \\ $(n=7)$}

(a)
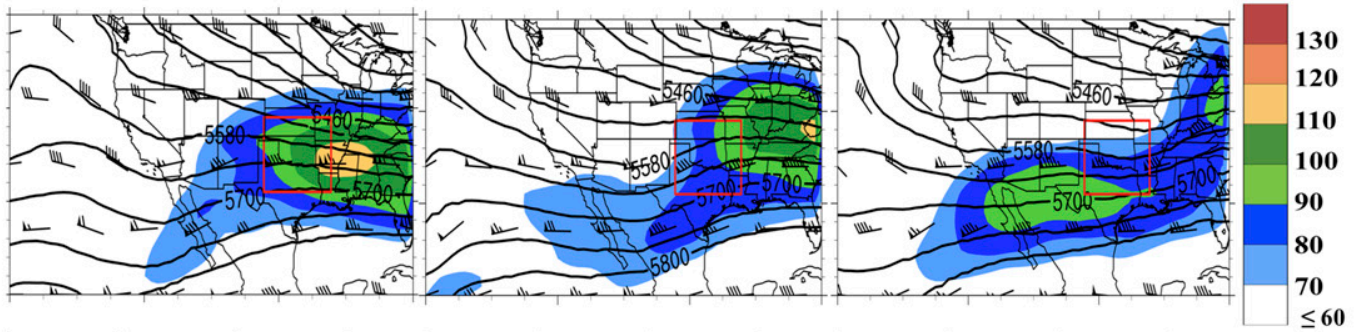

$|\vec{V}|$

(b)
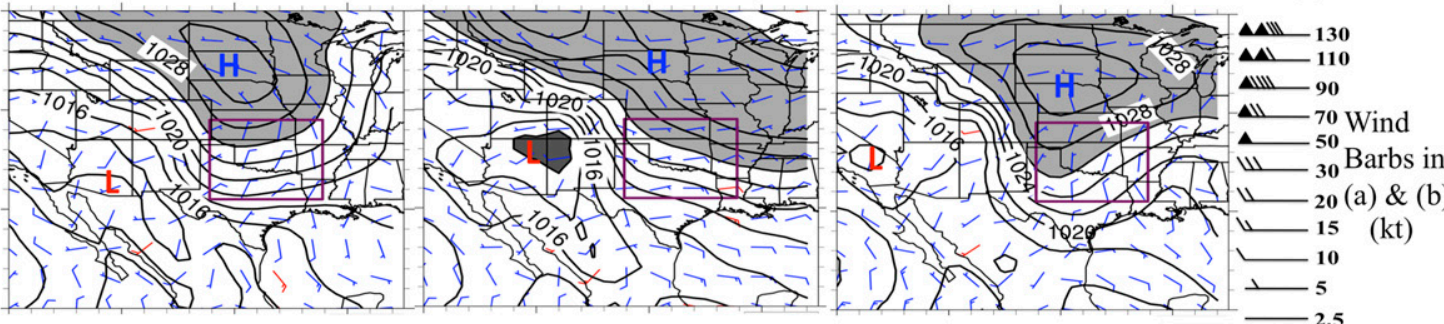

(c)
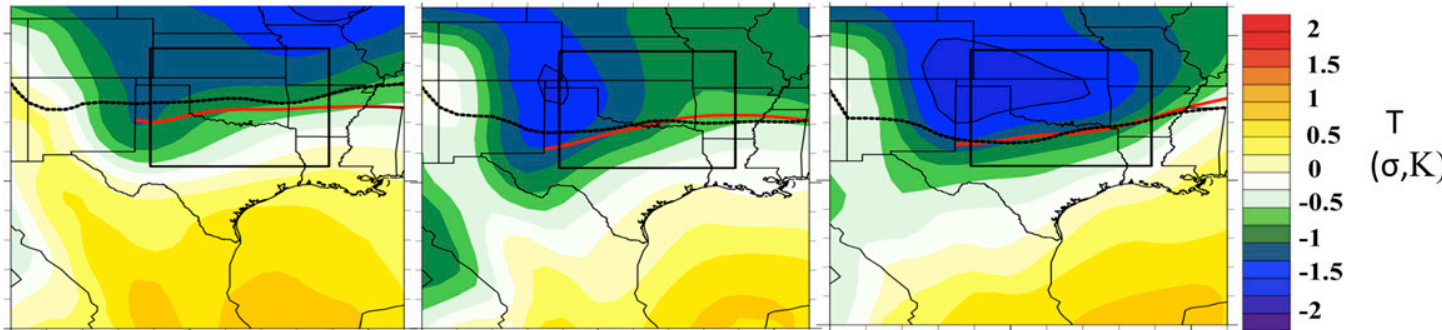

(d)
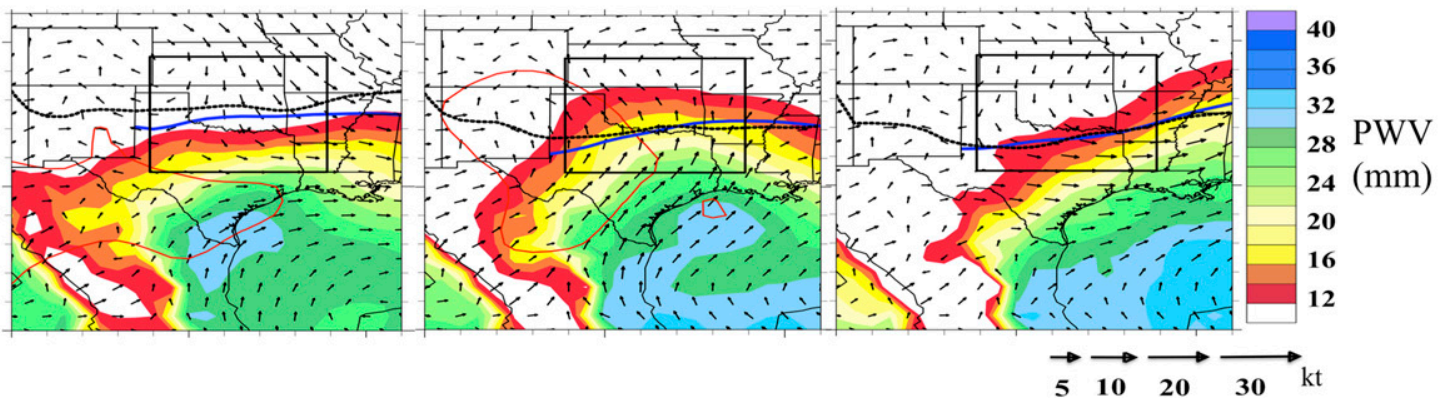

FIG. 13. As in Fig. 7, but for snow pattern 3. 


\section{Composite Ice Pattern 2 $(\mathrm{n}=6)$}

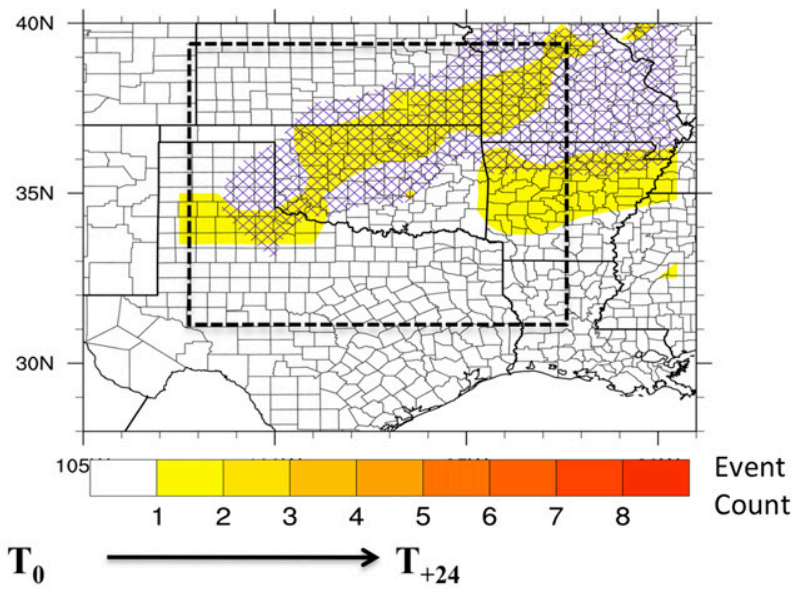

(a)
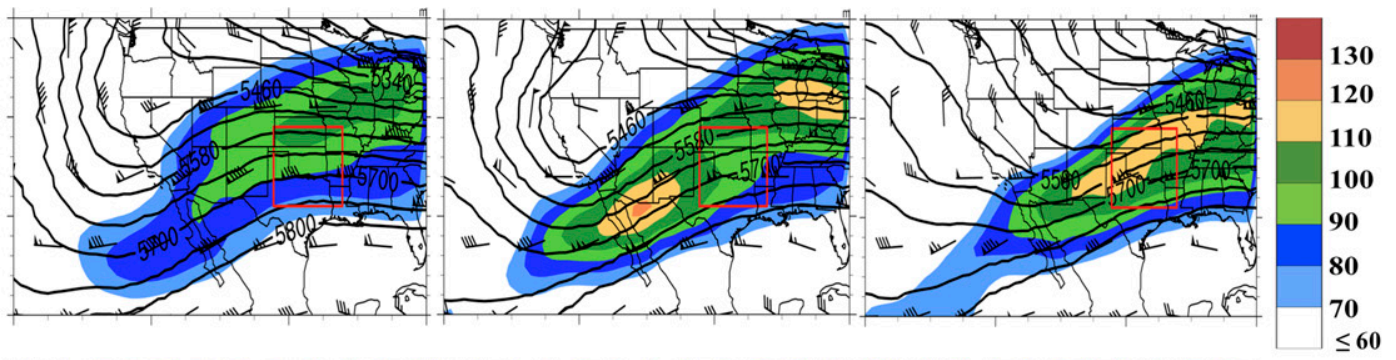

(b)
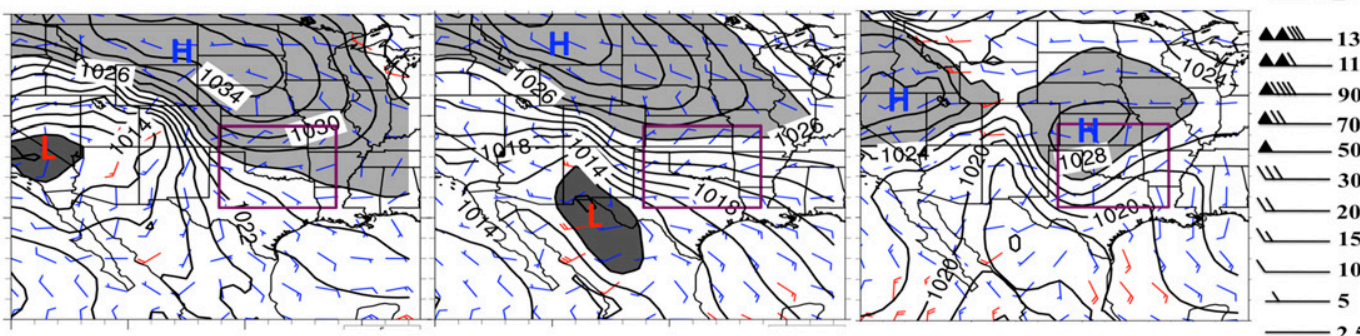

(c)
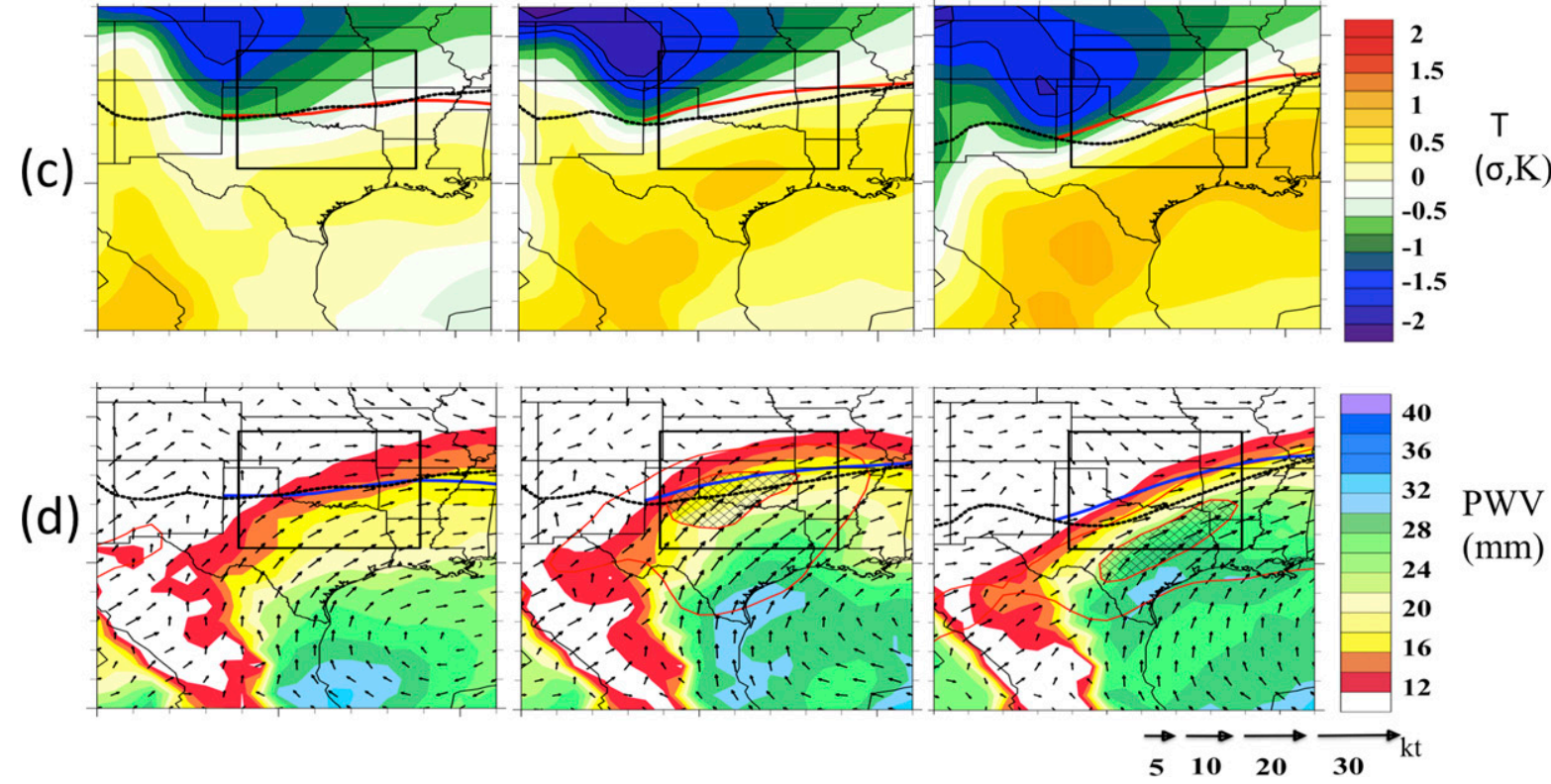

FIG. 14. As in Fig. 6, but for ice pattern 2. 


\section{Composite Ice Pattern 3 $(n=7)$}

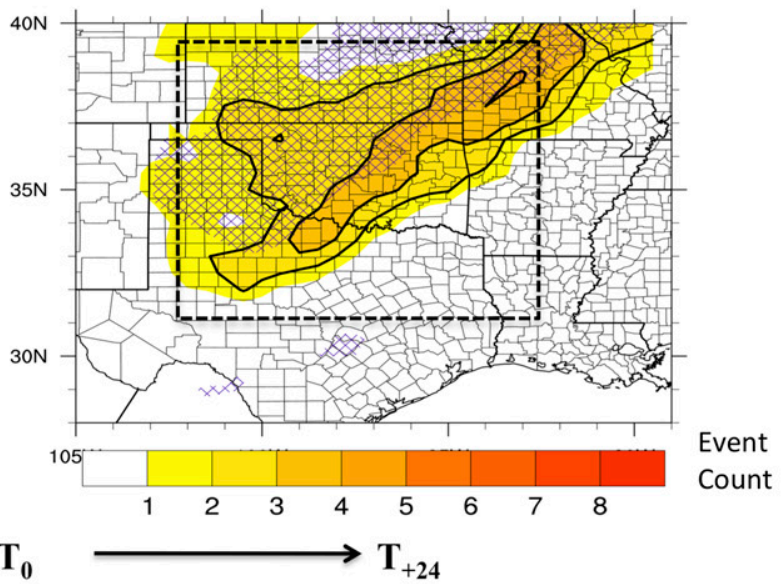

(a)
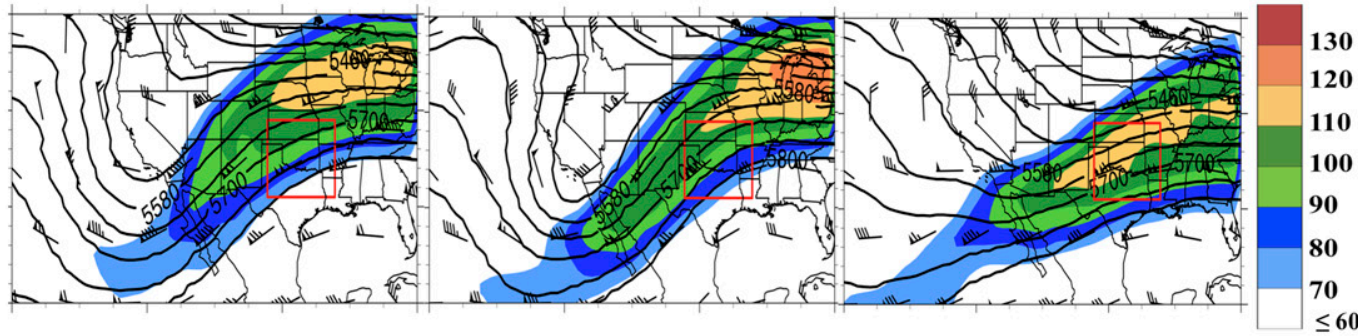

(b)
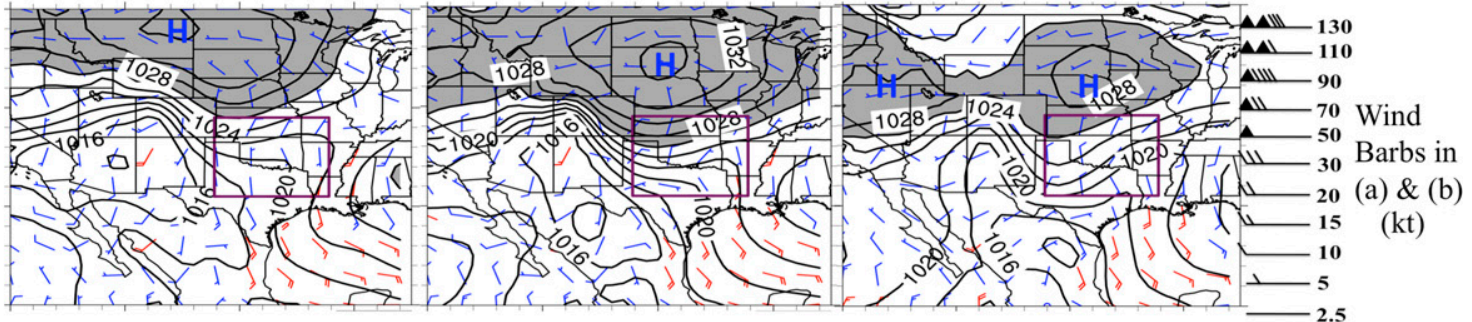

(c)
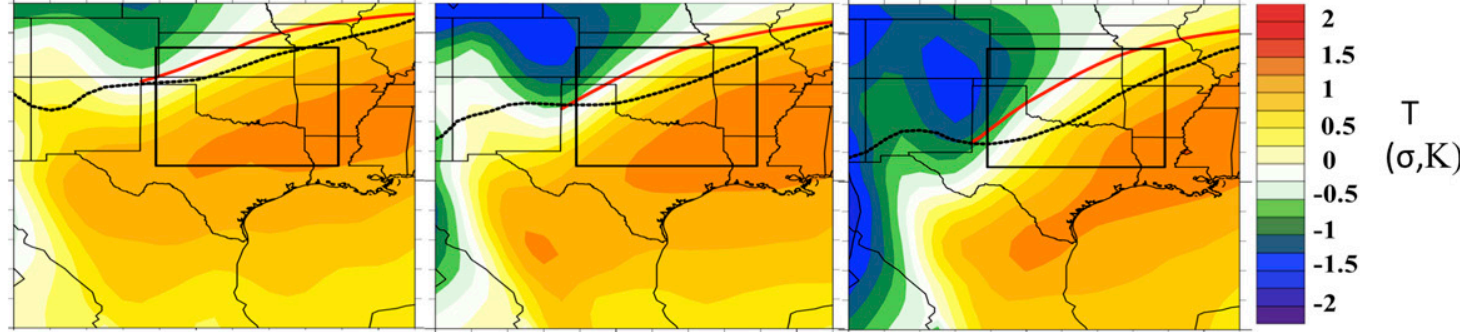

(d)
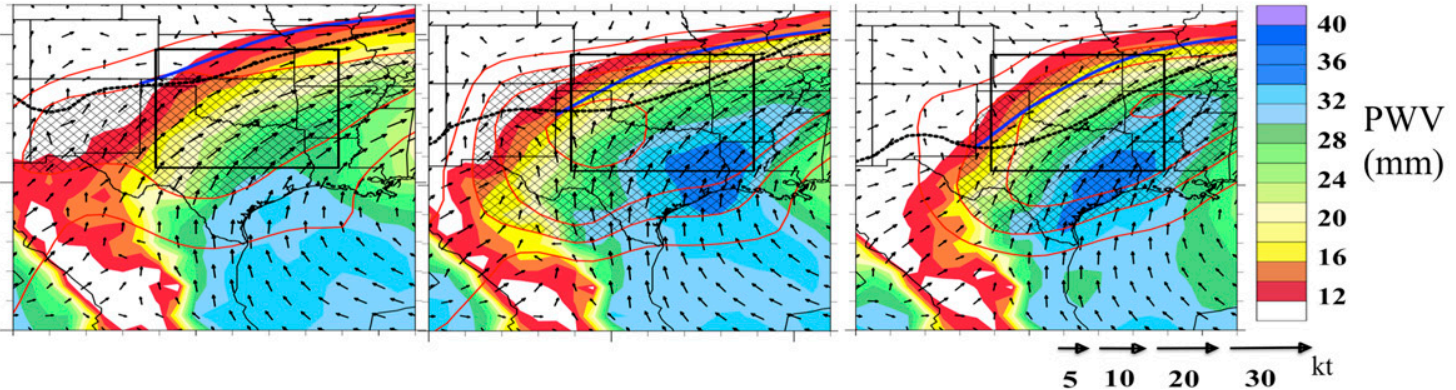

FIG. 15. As in Fig. 6, but for ice pattern 3 . 
temperature gradient, albeit the magnitude of the lowlevel temperature anomalies remains less than $+0.5 \sigma$. Moisture anomalies are also not notable, with a maximum near $T_{0}$ associated with northward advection from the GOM (Fig. 9d). The composite axis of freezing precipitation (Figs. 9c,d) suggests a generally narrow corridor for ice; however, individual events vary markedly, as evidenced by the spatial spread depicted in Fig. 9 (top). By $T_{+24}$, the greatest moisture and temperature anomalies have moved to the east and the upper trough resides near or over the domain. Wind and pressure fields (Fig. 9b) do not indicate consensus for surface low development, but pressure falls occur ahead of the trough $\left(T_{0}, T_{+24}\right)$.

Snow pattern 5 (Fig. 10) depicts a composite trough in the western United States over Baja California, bounded by northwesterly flow over the northern states and an upper jet to the east (Fig. 10a). By $T_{0}$, the southern trough axis has moved east to the domain periphery. The $850-\mathrm{hPa} 0^{\circ} \mathrm{C}$ isotherm lies near or south of its surface counterpart at $T_{-24}$ (Figs. 10c,d), with cool northwesterly flow in the lower troposphere. By $T_{0}$, warm air builds into the southern states (Fig. 10c), with CAA to the west behind a developing surface low pressure center (Fig. 10b). A brief window for ice is possible, but the location depicted in Figs. 10c and 10d resides north of the deeper moisture and is further limited by cooling aloft $\left(T_{+24}\right)$. While visually similar in evolution to ice pattern 5 , the snow composite suggests a weaker meridional temperature gradient and a stronger surface low pressure.

\section{2) LOW-AMPLITUde TROUGH}

Corresponding patterns include ice pattern 4 and snow patterns 1 and 3. At $T_{-24}$, ice pattern 4 (Fig. 11) shows broadly zonal flow, a strong height (temperature) gradient across the contiguous United States (Figs. 11a,c), and $110-\mathrm{kt}$ (where $1 \mathrm{kt}=0.51 \mathrm{~m} \mathrm{~s}^{-1}$ ) jet streak between the SGP and the mid-Atlantic. A weak-amplitude trough is located west of California. The SLP analysis (Fig. 11b) shows a cold anticyclone moving into the plains and strong downgradient northwesterly winds. This cold air mass is reflected by particularly anomalous $(\leq-1.5 \sigma)$ temperatures in the central plains (Fig. 11c). By $T_{0}$, the trough amplifies and moves into the southwest United States, while the eastern jet strengthens. Temperatures south and east of the SGP are increased, with anomalies $\geq+0.75 \sigma$. In addition, WAA broadens the composite freezing precipitation zone, while associated PWV increases in the southern subregion (Fig. 11d) are related to low-level GOM moisture trajectories. The central-southern and southeastern subdomains most commonly experience freezing precipitation with this pattern type (Fig. 11, top), while snowfall may occur farther north. The most abundant moisture typically moves east of the domain by $T_{+24}$ (Fig. 11d). Precipitation is likely driven by WAA/isentropic forced ascent over the Arctic frontal boundary. However, a weak surface low develops in the composite as the trough moves east of the domain.

The similarities in the height field of snow pattern 1 (Fig. 12) to ice pattern 4 are contrasted largely by thermodynamic distinctions. The SLP field at $T_{-24}$ (Fig. 12b) shows high pressure moving southeastward over the plains into the far southern United States, with northwesterly surface flow. In ice pattern 4 , this zone of highest SLP is oriented more north-south (Fig. 11b). As a consequence, the snow pattern evidences cooler 850-hPa temperatures in the southeast (Fig. 12c), as well as unremarkable moisture anomalies (Fig. 12d). This deeper cooler air mass drives the composite freezing isotherm at this level south of the surface isotherm, precluding sustained freezing precipitation. Additionally, as the trough axis moves eastward over the ensuing $48 \mathrm{~h}$, the $850-\mathrm{hPa}$ cold anomalies intensify southward. Events associated with this pattern tend to have particularly low surface temperatures, with snow common in the central and northern domain (Fig. 12, top). Propagation of the composite trough axis is also faster with respect to ice pattern 4 , aided by a lack of strong upper-level flow upwind of the trough axis and an expansive 100-120-kt jet to the east (Fig. 12a).

The focus for ascent for the SGP in snow pattern 3 (Fig. 13) is a short-wave trough propagating over the southwestern states through $T_{-24}$ and $T_{0}$ (Fig. 13a). The SLP field (Fig. 13b) is dominated by southward-moving high pressure over the plains prior to the winter precipitation, and a subsequent reinforcement of Artic air in the composite by $T_{-24}$. This evolution suggests shallow cold air in place over the domain as precipitation develops; however, the composite position of the $850-\mathrm{hPa}$ and surface freezing isotherms (Fig. 13c) suggest the cold air mass is deep and/or the warmer air mass to the south is sufficiently cool to favor largely ice pellets or snow. Temperature and moisture in advance of the cold air over the southern states and GOM show weak positive departures (Figs. 13c,d), and the development of a well-defined and moist warm layer is restricted by 850-hPa trajectories indirectly sourced from the GOM, and from the Pacific. As in snow pattern 1 (Fig. 12), cooler air continues to filter into the far southern United States by $T_{+24}$. Figure 13 (top) indicates that the northern half of the domain is again favored for snow.

\section{3) BROAD WESTERN TROUGH}

Within the resolved composite patterns, two ice events (ice patterns 2 and 3 ) and no snow-dominant 
events lie in this category. Ice pattern 2 (Fig. 14) shows a long-wave trough extending southwestward into Baja California at $T_{-24} \mathrm{~h}$, propagating slowly eastward over the subsequent $48 \mathrm{~h}$ (Fig. 14a). A strong baroclinic zone is present at low levels over the domain, orientated southwest to northeast by $T_{0}$ as the trough amplifies (Fig. 14c). Formation and orientation of this zone is aided by an apparent pressure gradient channeling of cold air along and east of the Rocky Mountains (Fig. 14b), with this cooler air mass moving into the southern domain at $T_{-24}$. By $T_{0}$, the temperature gradient across this zone has increased, intensifying a southwesterly jet aloft. Air ahead of the trough and south of the cold air mass over the southern United States, is warm, but not highly anomalous $(+0.75 \sigma)$. Anticyclonic flow over the Gulf of Mexico transports warm moist air into the domain from the western GOM and southeastern Pacific, with PWV departures maximizing at $+1.5 \sigma$, with $20-30-\mathrm{kt}$ flow (Fig. 14d). The composite average potential freezing precipitation zone is orientated parallel to the upper jet axis and moves southeast with time. Locations of ice for constituent events (Fig. 14, top) vary and do not favor a particular subregion but, generally, are situated parallel to the upper-level steering flow.

Ice pattern 3 (Fig. 15) is an amplification of features associated with ice pattern 2 (Fig. 14). The western trough is more meridional, but also slow moving, extending south to Baja California at $T_{0}$ (Fig. 15a), with SLP indicating downgradient advection of Arctic air into the SGP (Fig. 15b). Positive temperature anomalies extend farther north over the eastern United States, associated with a pronounced midlevel ridge. Temperatures in the southern and eastern United States are the warmest of all patterns, with anomalies $\geq+1.25 \sigma$ (Fig. 15c), and a broader composite zone of freezing precipitation is more consistently clustered southwest to northeast through the center of the SGP (Fig. 15, top). PWV anomalies are significantly positive, exceeding $+3 \sigma$ over much of the domain (Fig. 15d).

Ice pattern 3 has been associated with severe icing within the region, with four significant events since 2000 corresponding to the pattern. Contributing factors include 1) the quasi-stationary frontal boundary, with slow-moving upper-level flow, leading to the potential for prolonged precipitation; 2) direct GOM moisture transport; 3) upper-level steering flow parallel to the front; and 4) high temperatures in the warm layer promoting higher moisture content, sustaining freezing rain at higher precipitation rates. Similar flow fields have been linked with warm season heavy precipitation (Higgins et al. 2011). Nonetheless, the magnitude of precipitation is controlled by forcing for ascent, with the upper dynamical lift typically low for these pattern types as the main trough is well to the west (e.g., Ressler et al. 2012). Sanders et al. (2013) noted enhanced ascent contributed by short-wave troughs ejected ahead of the main long wave for a similar synoptic composite, and there is also a contribution from the relative progression of the upper trough for each constituent event. Mesoscale forcing also can be important, including low-level frontogenesis (e.g., Rauber et al. 1994) and elevated potential instability.

\section{c. Antecedent environment: Distinguishing characteristics}

Prior composite daily average parameters were evaluated for days 3-5 before the day of $T_{0}$, as displayed in Fig. 16 (500-hPa geopotential height anomaly, SLP mean) and Fig. 17 (850-hPa temperature anomaly, wind vector mean). Caveats regarding sample size aside, negative geopotential height anomalies for ice are more pronounced over the western and northern United States, and with all but one pattern type indicating a broad positive anomaly over the eastern and northern Pacific extending northward to Alaska (Figs. 16b-e). Northwestward expansion of the eastern Pacific anticyclone is particularly evident for ice patterns $2-4$ and snow pattern 1 with a weakened or displaced Aleutian low. This circulation pattern may imply increased blocked flow over the Pacific Northwest, linked to the displacement of Arctic high pressure into the midlatitude United States (Mullens 2014). Southward propagation of Arctic air may also be promoted on the western edge of a departing trough or surface cyclone. By $T_{-24}$, the SLP distributions shown in panel b in Figs. 6-15 indicated that ice events (Figs. 6, 9, 10, 14, and 15) were concurrent with preexisting higher SLP over the northern and central tiers. A Student's Welch two-tailed $t$ test for unequal variance (using NCAR Command Language: not shown) was performed between all 33 ice and 42 snow events at $T_{-24}$, resulting in a significant positive difference for ice east of the Rocky Mountains. Because of the small sample size, an additional check was run to evaluate the bootstrapped SLP confidence intervals at each grid point (250 replications; not shown), also producing significance at the $95 \%$ level. The presence of Arctic high pressure over the plains in advance of a SGP winter storm is therefore a strong indicator of whether freezing precipitation is likely to occur. Comparing composite average SLP distributions to archetypes developed by Rauber et al. (2001) reveals that SGP freezing precipitation commonly corresponds to archetypes A, C, and $\mathrm{D}$ from that study. In cases where limited icing and pronounced snow occurred in conjunction with an Arctic high (e.g., snow patterns 1 and 3 ) the air mass 

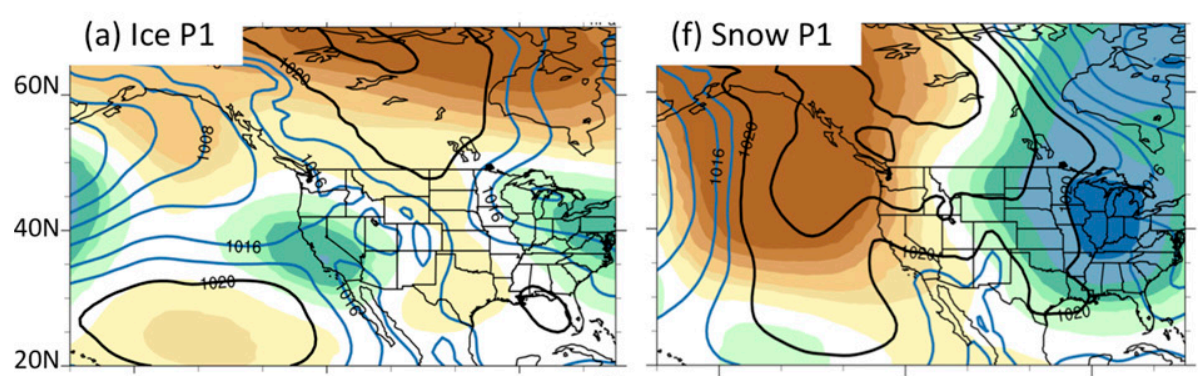

(b) Ice P2
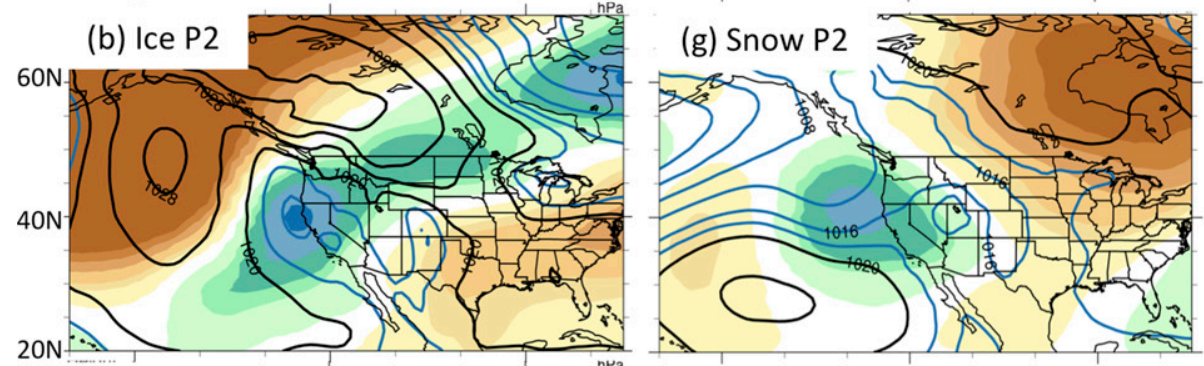

gpm
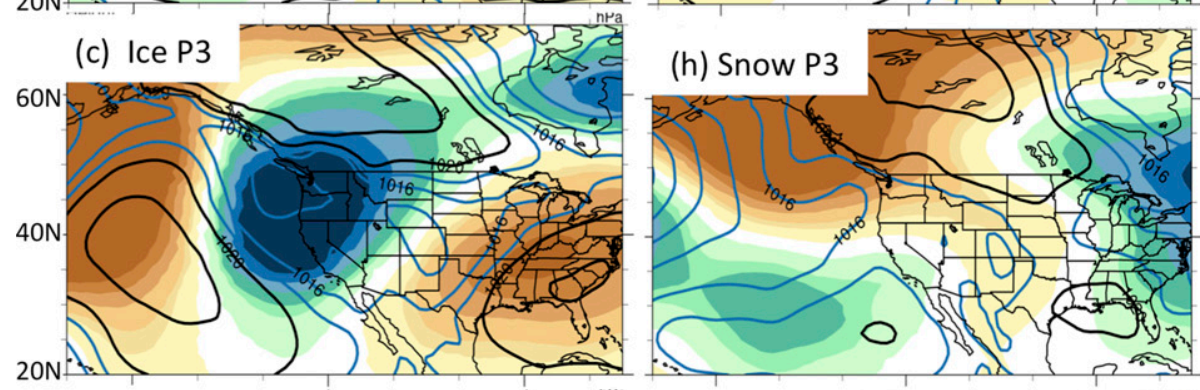

(d) Ice P4
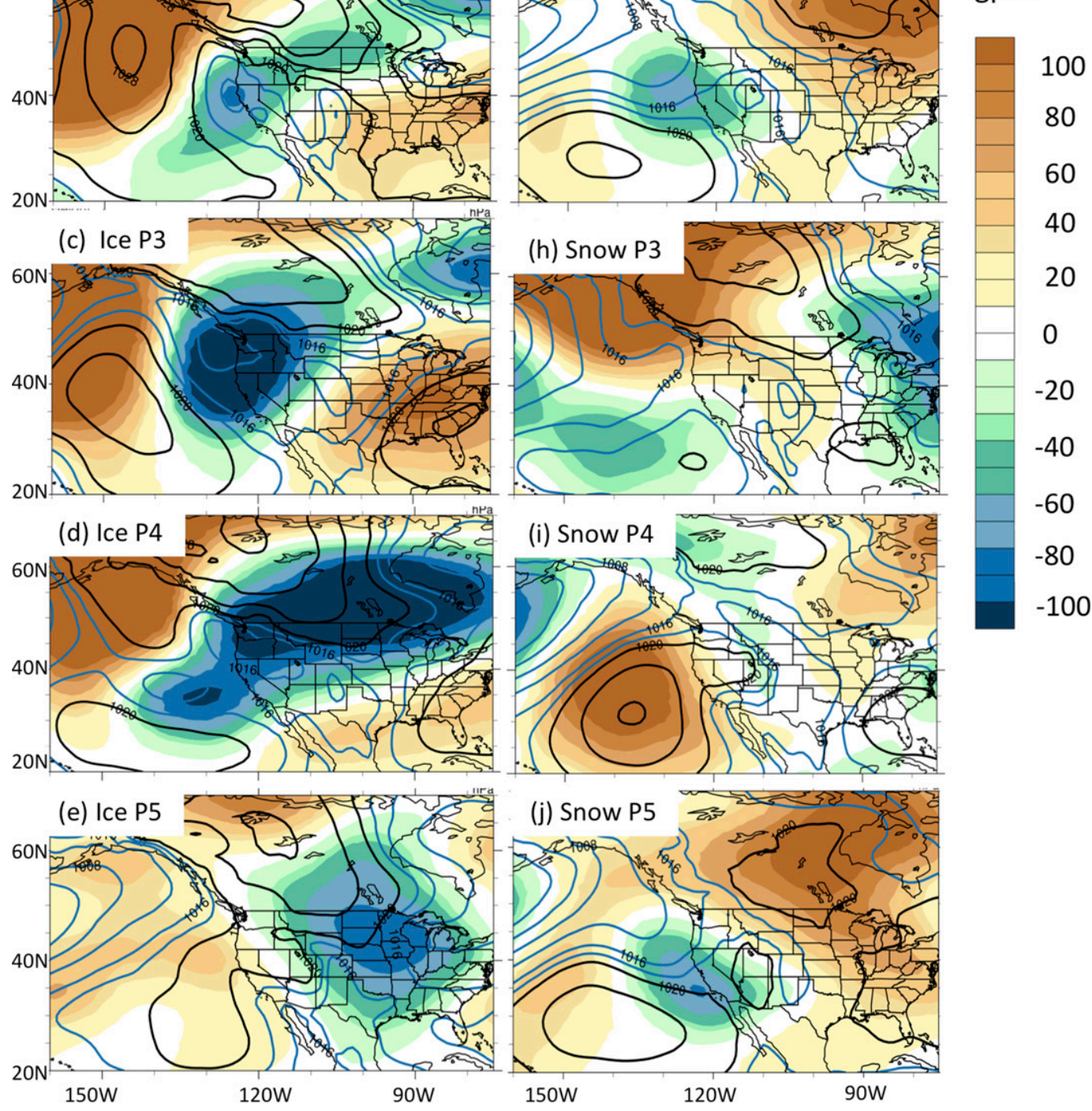

FIG. 16. Precursor environment as an average of daily data 3-5 days preceding the day of $T_{0}$ for each (left) ice and (right) snow pattern. Geopotential height anomalies (gpm) at $500 \mathrm{hPa}$ relative to NARR long-term daily climatology are shaded, while mean SLP is contoured, with values above (below) $1020 \mathrm{hPa}$ shown in black (blue).

was notably cool (e.g., Figs. 12 and 13), with the average preceding 3-5-day height and temperature anomalies suggesting a cool air mass over much of the eastern and southern United States under northwesterly low-level flow (Figs. 16 and 17). Resultantly, colder maritime subtropical air to the south and a deeper subfreezing air mass likely limited the potential for a well-defined warm layer. 

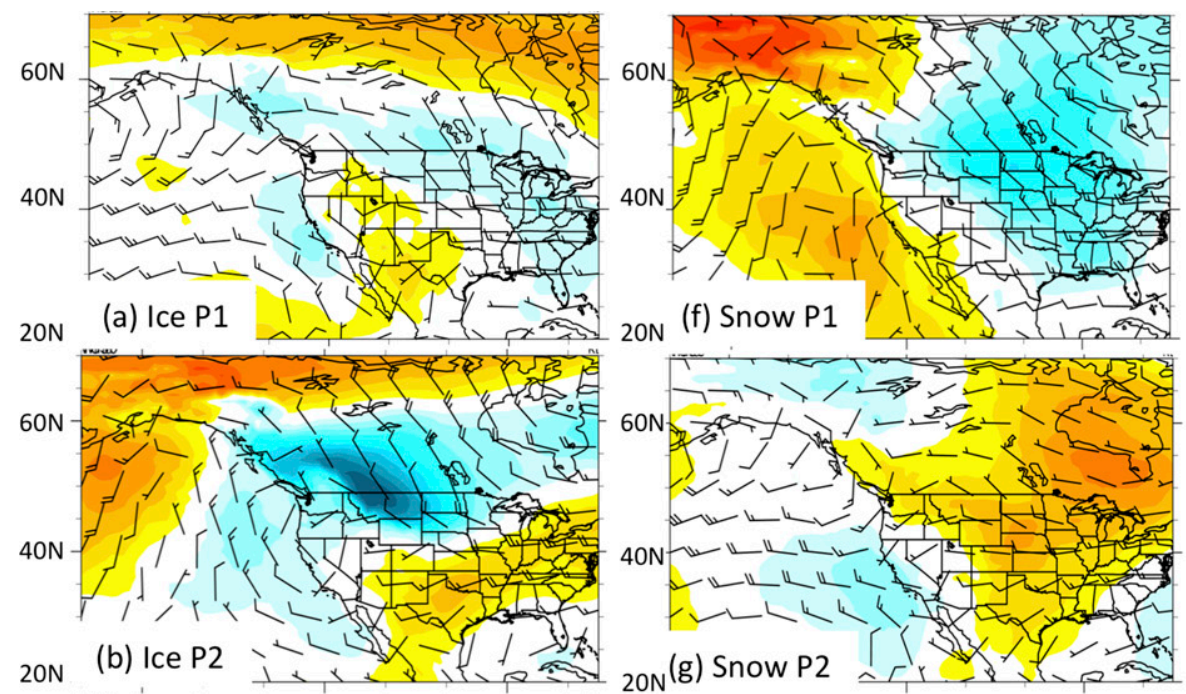

Anomaly

(K)
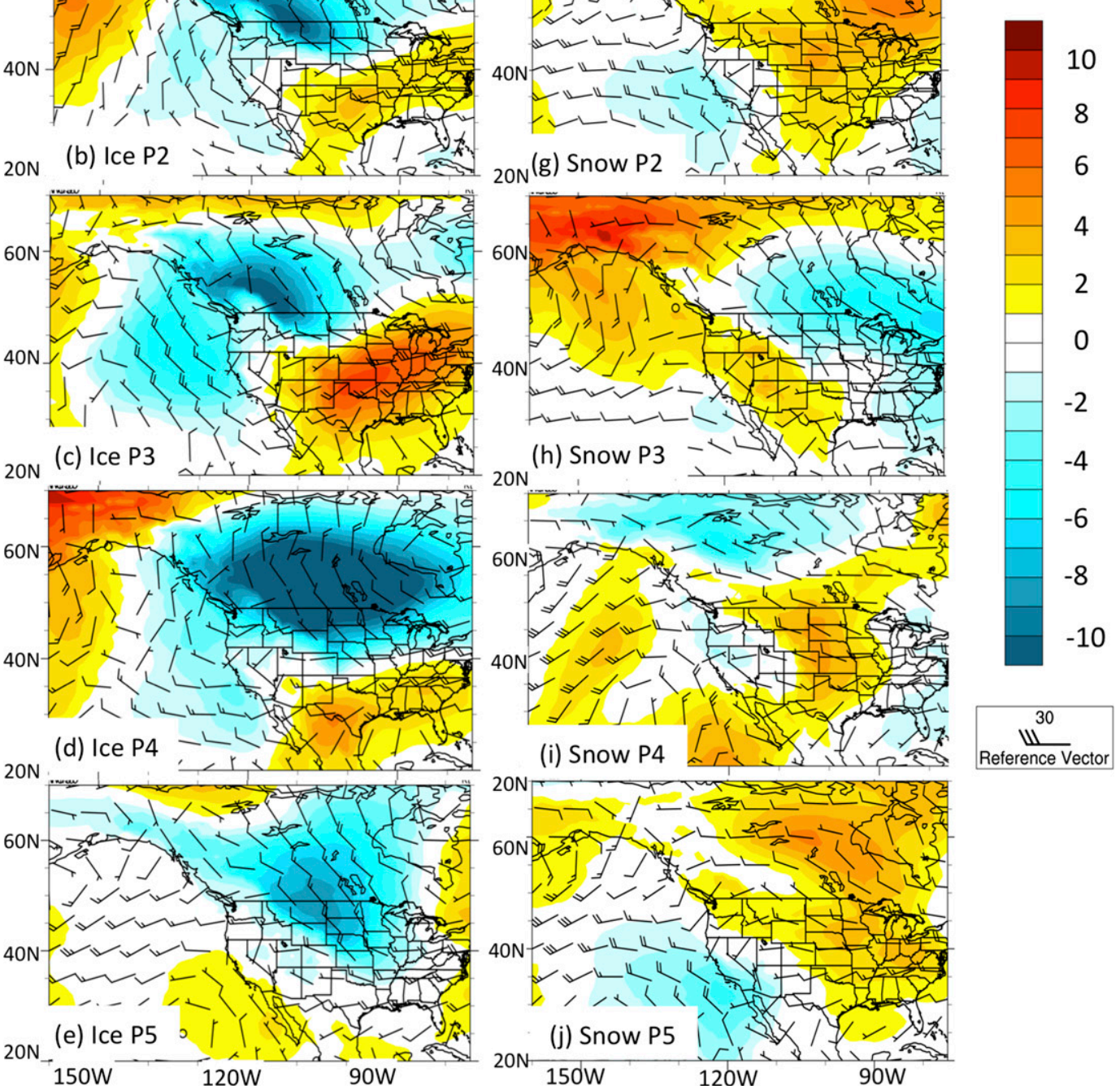

FIG. 17. Antecedent 850 -hPa air temperature anomalies (K; shaded) averaged over days $3-5$ before day $T_{0}$ for each (left) ice and (right) snow pattern. Mean 850-hPa vector winds are overlaid (barbs; kt).

Figure 17, in conjunction with the composite storm evolutions, demonstrates low-level thermal distinctions between composites and categories. The antecedent environment for ice generally showed an enhanced meridional temperature gradient over the contiguous states, particularly patterns $2-4$, which persisted as the winter storm evolved. Temperature anomalies over the northern tier were negative in every case, reflecting the incursion of Arctic air, while to the south neutral-toanomalously warm air was typically apparent with southerly and westerly component flow, weakest for ice patterns 1 and 5. Peak temperature anomalies ranged 


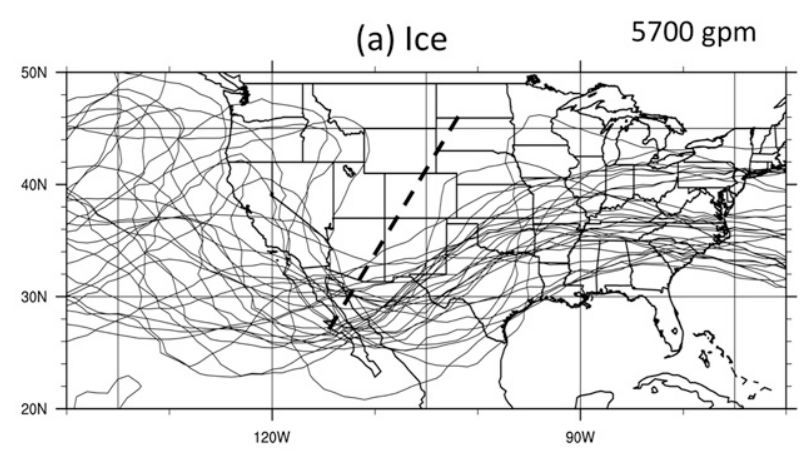

(b) Snow

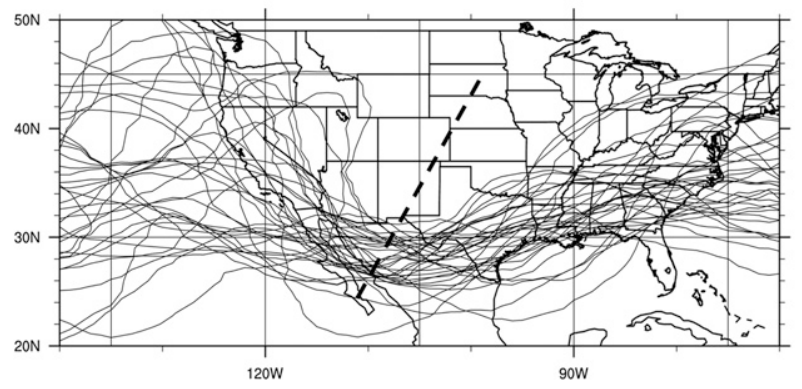

FIG. 18. Representation of the locations of troughs and ridges associated with (a) mixed-phase (ice) and (b) snow events at $T_{0}$ from the ice $(n=33)$ and snow $(n=42)$ datasets. Plotted contour is $5700 \mathrm{gpm}$. The dashed straight line is a subjective visual estimate of the most common location of the trough base and tilt.

from +2 to $+6 \mathrm{~K}$ over the long-term NARR daily climatology. Snow pattern antecedent environments did not tend to produce such a pronounced temperature gradient over the plains, with the possible exception of snow pattern 3 , being marked by cool air over the eastern and southern states (pattern 1), or broad warm anomalies over the plains (patterns 2, 4, and 5) with a tendency to westerly-northwesterly low-level winds for this period. A significance test was performed for bootstrapped (250 replications; not shown) mean 850-hPa temperatures at $T_{-24}$ and $T_{0}$, north and south of the freezing line, for a domain encompassing the SGP extended north (south) to $42^{\circ} \mathrm{N}\left(28^{\circ} \mathrm{N}\right)$ and east from $105^{\circ}$ to $90^{\circ} \mathrm{W}$. Pattern types were grouped by proximity and the amplitude of the major trough axis, and the presence of a surface cyclone versus a prominent baroclinic frontal boundary, yielding subgroups of ice patterns 1 and 5 (I1; $n=12$ independent events); ice patterns 2,3 , and $5(\mathrm{I} 2 ; n=17)$; snow patterns 2,4 , and $5(\mathrm{~S} 1 ; n=18)$; and snow patterns 1 and $3(\mathrm{~S} 2 ; n=$ 13). The I2 domain $850-\mathrm{hPa}$ temperatures above freezing were significantly higher $(95 \%$ confidence interval) than all other subgroups at $T_{0}$, but not $T_{-24}$ when compared with S1, while the 24-h (from $T_{-24}$ to $T_{0}$ ) coverage of above-freezing temperatures increased
$3 \%$, versus a $24-\mathrm{h}$ decrease for all other categories. Patterns I1, S1, and S2 did not evidence significant differences in temperature at either time, and their patterns of evolution were accompanied by expansion of subfreezing surface temperatures. S2 was statistically cooler than $\mathrm{I} 1$ and $\mathrm{S} 1$, but not when compared to I2. S1 showed the largest 24-h positive expansion of subfreezing air (30\%), followed by I1 $(12.5 \%)$.

Finally, this sample exhibited a tendency for the trough axis position of ice events at $T_{0}$ to lie, on average, to the west of that for snow (e.g., $\sim 59 \%$ ice, versus $27 \%$ snow, with the major trough axis base west of the Arizona-New Mexico border), which is illustrated by plotting the $5700-\mathrm{m}$ geopotential height contour in Fig. 18. Forcing for ascent in freezing precipitation therefore may be more commonly associated with frontal dynamics and temperature advection [e.g., "west composites"; Ressler et al. (2012)], while surface cyclone formation prior to $T_{0}$ is largely affiliated with snow-dominated systems, in conjunction with an upper trough in the domain vicinity. Conditions promoting mature surface cyclones in snow-dominant patterns possibly could be related to the low-level static stability in advance of the trough. We can contrast the weaker cyclone in composite ice pattern 1 (Fig. 6) to those of snow patterns 2 (Fig. 7) and 4 (Fig. 8). Within a quasigeostrophic framework, it is likely that in the former case, cyclone development is retarded because of nearsurface CAA, high static stability, and anticyclonic relative vorticity associated with the high pressure ridge. These act to oppose ascent; for example, low-level anticyclonic vorticity acts in contrast to cyclonic vorticity aloft and reduces column stretching, while height rises in the warm layer oppose the height falls from the approaching trough (Bluestein 1993, p. 169).

\section{Summary}

This study provided a regional spatial and synoptic climatology of freezing precipitation and snow over the U.S. SGP, by developing and analyzing a 1993-2011 regional case study database of winter weather. Despite the small sample size and limited temporal range, some insightful information has resulted from this analysis. Domain spatial distribution suggests that accumulating snow is most common over the north, west, and elevated terrain of this region. In contrast, freezing precipitation occurs most frequently over the central and northeastern domains. While the seasonal range of freezing precipitation is primarily December-February, snowfall can occur throughout the cool season (NovemberMarch). Nonetheless, winter weather of either category remains comparatively infrequent compared to other 
TABLE A1. List of events forming the dataset for ice used in the PRPC analysis. Highlighted are time $T_{0}$, the patterns each case study was found to correspond with (where 0 indicates the event did not correlate with any of the resolved patterns from the four PRPCs), and approximate locations impacted (state abbreviations where C indicates central). Using a combination of information, including Storm Data and NCEI local climate summaries, durations and liquid precipitation equivalents (LPEs) are estimated. Durations are estimates of the length of time precipitation occurred within the domain boundary, based on measurements from various station sites. LPE is evaluated from surface observations for several major stations over the impacted area, and the uppermost value is used, to provide an upper (station estimated) threshold for each event. In cases where events are in two or more pattern groups, the highest correlated pattern (if applicable) is denoted by a plus sign $\left(^{+}\right)$.

\begin{tabular}{|c|c|c|c|c|}
\hline Event time and date $\left(T_{0}\right)$ & Type (ice) & Areas impacted & $\begin{array}{c}\text { Approx duration } \\
\text { of event }(\mathrm{h}) \text { from } \\
\text { representative station }\end{array}$ & $\begin{array}{c}\text { Approx max } \\
\text { LPE (in.) from } \\
\text { representative station }\end{array}$ \\
\hline 2100 UTC 1 Jan 1993 & 6 & S KS, W-C-E OK, N TX & 24 & 0.25 \\
\hline 1200 UTC 18 Jan 1993 & 2 and $7^{+}$ & $\mathrm{N}-\mathrm{C}$ AR, N TX & 30 & 0.70 \\
\hline 1500 UTC 25 Nov 1993 & 0 & E-S OK, N TX & 15 & 0.22 \\
\hline 0000 UTC 9 Feb 1994 & 4 & SE OK, SW AR, NE TX & 30 & 0.55 \\
\hline 1200 UTC 6 Jan 1995 & 8 & C-N AR, S MO, E OK & 6 & 0.55 \\
\hline 0000 UTC 2 Feb 1996 & 4 and $5^{+}$ & NE TX, NW LA, S AR & 12 & 0.39 \\
\hline 1800 UTC 24 Nov 1996 & 1 & N TX, S OK & 6 & 0.60 \\
\hline 1200 UTC 8 Jan 1997 & $1^{+}$and 5 & SE OK, N TX, S-C AR & 24 & 0.45 \\
\hline 0600 UTC 15 Jan 1997 & 7 & S-SE OK, NE TX, S-C AR & 30 & 0.56 \\
\hline 1200 UTC 4 Jan 1998 & 6 & S KS & 12 & 1.10 \\
\hline 1200 UTC 16 Mar 1998 & 1 & N TX Panhandle, S KS & 12 & 0.46 \\
\hline 1800 UTC 20 Dec 1998 & 2 & N OK, S KS, SW MO & 38 & 0.35 \\
\hline 0000 UTC 8 Jan 1999 & 5 & NW TX, SW and C OK, C AR & 15 & 0.10 \\
\hline 0000 UTC 13 Dec 2000 & 4 & NC TX, S OK, SW AR & 24 & 0.65 \\
\hline 0000 UTC 26 Dec 2000 & 1 & N TX, S OK, SW-C AR & 42 & 1.07 \\
\hline 1800 UTC 28 Jan 2001 & 1 & SC-NC OK, NE OK & 24 & 1.00 \\
\hline 1200 UTC 27 Feb 2001 & 3 and $7^{+}$ & $\mathrm{C}$ and SE KS & 12 & 0.24 \\
\hline 1800 UTC 28 Nov 2001 & $1^{+}$and 4 & N TX & 12 & 0.29 \\
\hline 000030 UTC Jan 2002 & 3 & S-SE KS, N-C and W-C OK OK & 36 & 1.10 \\
\hline 0000 UTC 4 Dec 2002 & 1 & $\mathrm{C}$ and $\mathrm{NE} \mathrm{OK}, \mathrm{N}$ AR & 15 & 0.86 \\
\hline 1200 UTC 26 Feb 2003 & 5 & C TX, E OK, S AR & 10 & 0.30 \\
\hline 1800 UTC 4 Jan 2005 & $2,3^{+}$and 6 & N OK, S KS & 18 & 1.82 \\
\hline 1800 UTC 19 Feb 2006 & 2 & C-NE OK, W-C AR & 12 & 0.36 \\
\hline 1200 UTC 19 Dec 2006 & 3 and $7^{+}$ & SW KS, TX Panhandle & 24 & $<0.5$ \\
\hline 1200 UTC 30 Dec 2006 & 1 & C-E TX Panhandle & 24 & 0.25 \\
\hline 0000 UTC 13 Jan 2007 & 3 & $\mathrm{C}$ and E-NE OK, SW MO, SE KS & 24 & 2.58 \\
\hline 1200 UTC 14 Jan 2007 & 3 & C-E OK, N-C TX C-S MO & 24 & 0.79 \\
\hline 1200 UTC 9 Dec 2007 & 3 & SW-C-NE OK, SW MO, NW AR & 48 & 2.05 \\
\hline 0600 UTC 22 Jan 2008 & $2^{+}$and 7 & SW, C-NE OK, NW AR & 6 & 0.25 \\
\hline 1800 UTC 15 Feb 2008 & 5 and 7 & $\mathrm{C}$ and SE OK & 24 & $<0.5$ \\
\hline 1200 UTC 23 Dec 2008 & 4 & SE OK, E KS, C AR & 10 & $<0.25$ \\
\hline 1800 UTC 26 Jan 2009 & 2 & C-E OK, C-N AR & 30 & 1.26 \\
\hline 1200 UTC 28 Jan 2010 & 5 & SW-C OK, E TX Panhandle & 12 & 1.06 \\
\hline
\end{tabular}

regions of the country $\left(<2-4\right.$ days $\left._{\mathrm{yr}^{-1}}\right)$. The domain thermodynamics of freezing rain, freezing drizzle, ice pellets, and snow are consistent with past studies. Freezing precipitation is typically associated with a warm layer, the vertical extent and magnitude of which can be pronounced for ice storms in this region.

The results of a rotated principal component and composite analysis for ice- and snow-dominant SGP events yielded distinctions between composite mixed-phase (ice) storms versus snowstorms. The primary differences were typically at the low levels. The near-surface air mass over the plains in advance of an upper trough (e.g., Fig. 14) often is cooler for ice, bounded by a warmer air mass to the south. This distribution promotes increased baroclinicity, as evidenced by the frequently stronger and broader upper-level jet over the central and eastern United States for ice patterns $1-5\left(T_{-24}, T_{0}\right)$. This thermal boundary demarcates Arctic and maritime subtropical air, which is commonly associated with a quasi-stationary front. Evidence for the preponderance of an Arctic (cold) air mass was identified by examination of the SLP at $T_{-24}$, where all composite "ice" storms showed evidence of a surface high over the northern and central plains prior or during event onset. This result is found to be statistically distinct from snow-dominant cases. Moreover, near-surface ageostrophic wind and isobaric troughing over the 
TABLE A2. As in Table A1, but for snow events. Snow amounts are estimated in a similar fashion to the LPE estimates. When the estimate is uncertain (e.g., snowfall bands outside of measurement locations), measurements from the Storm Data archive are used. As in Table A1, upper thresholds are shown below. Missing snow accumulation information is shown as a dash.

\begin{tabular}{|c|c|c|c|c|}
\hline $\begin{array}{l}\text { Event time and } \\
\quad \text { date }\left(T_{0}\right)\end{array}$ & $\begin{array}{c}\text { Type } \\
\text { (snow) }\end{array}$ & Locations impacted & $\begin{array}{l}\text { Approx duration } \\
\text { of event }(\mathrm{h}) \text { from } \\
\text { representative } \\
\text { station }\end{array}$ & $\begin{array}{l}\text { Approx LPE } \\
\text { (snowfall) (in.) from } \\
\text { representative } \\
\text { station }\end{array}$ \\
\hline 0000 UTC 9 Jan 1993 & 0 & OK-TX Panhandle, NW, NC and C OK, S KS & 30 & $0.30(6.3)$ \\
\hline 0600 UTC 15 Feb 1993 & $1,3^{+}$, and 5 & NW-NE OK, S-SW KS, S MO & 48 & $0.77(11.2)$ \\
\hline 1200 UTC 25 Feb 1993 & 3 & N OK, SW-S KS, SW MO & 24 & $0.69(12.0)$ \\
\hline 1800 UTC 8 Mar 1994 & 0 & $\begin{array}{l}\text { N OK, OK Panhandle, E, and } \\
\text { C OK, C and N AR }\end{array}$ & 24 & $1.75(12.9)$ \\
\hline 0000 UTC 19 Jan 1995 & 2 & S MO, SE KS & 18 & $0.57(8.0)$ \\
\hline 1800 UTC 12 Feb 1995 & 1 and $3^{+}$ & $\begin{array}{l}\text { SW-E OK-TX Panhandle, } \\
\text { NW-C OK, SW-SC KS }\end{array}$ & 12 & $0.21(5.1)$ \\
\hline 1200 UTC 1 Mar 1995 & 3 & SW KS, NC OK & 30 & $0.50(9.2)$ \\
\hline 0600 UTC 7 Mar 1995 & 0 & SW KS, N OK & 24 & $0.22(4-6)$ \\
\hline 1200 UTC 18 Dec 1995 & $2^{+}$and 5 & $\begin{array}{l}\text { NW OK, S KS, N-C and NE } \\
\text { OK-TX Panhandle }\end{array}$ & 21 & $0.40(5.0)$ \\
\hline 0900 UTC 2 Jan 1996 & 4 & NE OK, N AR, SW MO & 24 & $1.20(8.2)$ \\
\hline 1200 UTC 18 Jan 1996 & 6 & NW OK, C KS & 12 & $1.27(4)$ \\
\hline 0600 UTC 2 Feb 1996 & 1 & C OK, C AR and N TX & 18 & $0.15(6-9)$ \\
\hline 1200 UTC 8 Jan 1997 & 4 & W, C, and E OK; SE KS; N AR; S MO & 24 & $0.80(9.0)$ \\
\hline 1200 UTC 8 Jan 1998 & 0 & S-SE KS, SW MO & 18 & $0.47(4.2)$ \\
\hline 0000 UTC 8 Mar 1998 & 2 & S-SW KS, N TX Panhandle & 18 & $0.66(9.3)$ \\
\hline 1200 UTC 12 Mar 1999 & 2 & TX-OK Panhandle, NW OK, SW KS & 24 & $1.81(13.0)$ \\
\hline 2100 UTC 26 Jan 2000 & 0 & W, C, and E OK; S KS; N AR; N TX & 21 & $0.80(8.9)$ \\
\hline 0000 UTC 13 Dec 2000 & 3 & N OK, N AR, SW MO & 24 & $1.05(7.3-14.2)$ \\
\hline 0900 UTC 31 Dec 2000 & 0 & E OK, NW AR & 21 & $0.13(4-6)$ \\
\hline 0000 UTC 5 Dec 1999 & 2 & $\mathrm{~S} \mathrm{KS}, \mathrm{N}-\mathrm{C} \mathrm{OK}$ & 9 & $0.63(4.5)$ \\
\hline 1200 UTC 16 Jan 2001 & 5 & NW OK, TX Panhandle, S KS & 27 & $0.24(4-10)$ \\
\hline 1800 UTC 28 Nov 2001 & 2 & W-C OK, SW-C MO & 15 & $-(6.0-8.0)$ \\
\hline 1800 UTC 5 Feb 2002 & 2 & SE TX Panhandle, NW TX and C OK, N AR & 18 & $0.24(2-6)$ \\
\hline 0000 UTC 2 Mar 2002 & 1 & $\mathrm{~N}-\mathrm{C}$ OK, NE-E OK & 21 & $0.70(6.4)$ \\
\hline 0000 UTC 4 Dec 2002 & 3 & S KS; N, W, C OK; S MO & 24 & $0.76(7.6)$ \\
\hline 0000 UTC 24 Dec 2002 & 2 and $5^{+}$ & $\mathrm{N} \mathrm{OK}$ and $\mathrm{S} \mathrm{KS}$ & 24 & $0.80(7.5)$ \\
\hline 1200 UTC 9 Feb 2003 & 1 & $\mathrm{~N}-\mathrm{C}, \mathrm{N}, \mathrm{N}-\mathrm{E} \mathrm{OK} ; \mathrm{S} \mathrm{KS}$ & 10 & $0.20(4-10)$ \\
\hline 1200 UTC 23 Feb 2003 & 0 & $\mathrm{~S} \mathrm{KS}, \mathrm{N}$ and $\mathrm{C} \mathrm{OK}, \mathrm{C}$ and NAR, $\mathrm{S} \mathrm{MO}$ & 72 & $0.86(11.5)$ \\
\hline 2100 UTC 12 Dec 2003 & 0 & N OK, SW KS, N AR & 15 & $0.37(7.3)$ \\
\hline 1800 UTC 13 Nov 2004 & $2^{+}$and 5 & TX Panhandle & 12 & $0.61(4.4)$ \\
\hline 1200 UTC 22 Dec 2004 & $1^{+}$and 4 & $\mathrm{C}-\mathrm{S}$ OK, C-N AR & 12 & $0.15(2-6)$ \\
\hline 1800 UTC 28 Jan 2005 & $2^{+}$and 3 & C-N OK, S KS, TX Panhandle & 18 & $0.35(3.1)$ \\
\hline 1200 UTC 17 Dec 2005 & 0 & SW-SE KS & 24 & $0.52(5.6)$ \\
\hline 1800 UTC 9 Jan 2006 & 0 & $\begin{array}{l}\text { TX Panhandle, W and C OK, } \\
\text { SW-SE KS, NE OK }\end{array}$ & 14 & $-(3.0-8.0)$ \\
\hline 0000 UTC 30 Nov 2006 & 0 & SW-SE KS, N and C OK, SW MO & 21 & $1.14(10.4)$ \\
\hline 1200 UTC 20 Jan 2007 & 5 & $\mathrm{~S} \mathrm{KS}, \mathrm{N}$ and $\mathrm{C} \mathrm{OK}$ & 18 & $0.40(5.2)$ \\
\hline 1800 UTC 31 Jan 2008 & 6 & SE KS, N OK, N AR, S MO & 18 & $0.28(4.0-6.0)$ \\
\hline 1800 UTC 3 Mar 2008 & 4 & E OK, N and C AR, SW MO (Ozarks) & 12 & $-(4.0-8.0)$ \\
\hline 1800 UTC 6 Mar 2008 & 4 & E OK, C AR, N TX & 12 & 0.93 (up to 12.0 ) \\
\hline 0000 UTC 25 Dec 2009 & 4 & $\mathrm{C} \mathrm{OK}, \mathrm{N}-\mathrm{E}$ OK, and N AR & 12 & $0.95(13.5)$ \\
\hline 0000 UTC 1 Feb 2011 & 1 & $\mathrm{C}$ and $\mathrm{N}$ OK, NE OK and NW AR, SW MO & 12 & $1.00(13.2)$ \\
\hline 0000 UTC 9 Feb 2011 & 1 & $\mathrm{C}$ and $\mathrm{N}$ OK, NE OK and NW AR, SW MO & 15 & $0.40(6.0)$ \\
\hline
\end{tabular}

western high plains suggest the potential for topographically aided cold-air damming, accelerating the southward propagation of shallow cold air to the domain. The composite 850-hPa flow revealed that moisture for winter storms is typically sourced from the GOM and the Pacific during freezing precipitation, with the most-abundant moisture associated with a warm ridge over the southeastern United States and anticyclonic southerly winds over the western GOM. The 500-hPa height composites suggest that snow patterns are associated with more amplified upper flow and surface cyclone development. Nonetheless, snow also occurs north of an elongated 
surface front and within a deeper cold air mass under more zonal regimes. Freezing precipitation occurs mostly north of a quasi-stationary surface front, but is also observed northeast of the weak surface low pressure.

The common southwest-northeast orientation of freezing precipitation observed in the synoptic analysis (e.g., Figs. 7,12, and 13) corresponds to the spatial climatology (Fig. 2b). However, it is suspected that, as a result of event infrequency, the spatial distribution is weighted toward long-duration episodes of icing, such as ice pattern 3 . This pattern is a common ice storm circulation for the central and northeastern SGP, whereas ice pattern 4 is common in the south and east. For snow, moisture potential is greatest north of developing surface cyclones, while duration is enhanced for the weaker-forced frontal systems. Advection and thermal changes associated with snow-dominant systems suggest persistent cooling of the vertical profile.

The results shown here can assist forecasters in forming a conceptual pattern-based understanding of the precursor environment and evolution of weather systems producing freezing and frozen precipitation in the SGP. The patterns are a large subset of common flow fields, and can provide vital information on possible durations and intensities of approaching winter systems displaying similar characteristics. However, actual storm events do not necessarily evolve as shown here and, often, are a blend of pattern types or incorporate evolutions not addressed by this work. Furthermore, the positioning of the $0^{\circ} \mathrm{C}$ isotherm is also a critical forecast challenge that was not investigated here. Because forecasting of precipitation type is a critical aspect of winter weather, this study is intended to assist forecasters in interpreting conditions that favor a specific precipitation phase from medium-range guidance. Future work will examine additional case studies to confirm and expand upon the findings of this study.

Acknowledgments. This research was part of EDM's dissertation research, and was supported by the Atmospheric System Research (ASR) program of the U.S. Department of Energy (Grant DE-FGDZ-05ER64062), as a contribution from the ARM Southern Great Plains site scientist team. Additional funding was from the NOAA-University of Oklahoma (Cooperative Agreement NA080AR4320904). The authors thank Dr. Michael Richman for his assistance in the statistical techniques used in this paper. Most statistical methods were implemented using $\mathrm{R}$ program (https://www.r-project. org), along with visualizations using $\mathrm{R}$, and NCAR Command Language (http://www.ncl.ucar.edu). This work has benefited from the contributions of Dr. Peter Lamb, who sadly passed away before its competition.

\section{APPENDIX}

\section{Winter Storm Event Dataset}

Tables A1 and A2 provide details on the ice and snow events that create the dataset used in this analysis.

\section{REFERENCES}

Ackerman, T. P., and G. M. Stokes, 2003: The Atmospheric Radiation Measurement Program. Phys. Today, 56, 38-44, doi:10.1063/1.1554135.

Baldwin, J., 1973: The Climates of the United States. NOAA, 113 pp. [NTIS COM-74-11708/6.]

Bennett, I., 1959: Glaze: Its meteorology and climatology, geographical distribution, and economic effects. Environmental Protection Research Division Tech. Rep. EP-105, Headquarters, U.S. Army Quartermaster, Research and Engineering Command, Natick, MA, 217 pp. [NTIS AD-216668.]

Bernstein, B. C., 2000: Regional and local influences on freezing drizzle, freezing rain, and ice pellets. Wea. Forecasting, 15, 485508, doi:10.1175/1520-0434(2000)015<0485:RALIOF >2.0.CO;2.

Blackmon, M. L., 1976: A climatological spectral study of the $500 \mathrm{mb}$ geopotential height of the Northern Hemisphere. J. Atmos. Sci., 33, 1607-1623, doi:10.1175/1520-0469(1976)033<1607: ACSSOT $>2.0 . \mathrm{CO} ; 2$

Bluestein, H. B., 1993: Observations and Theory of Weather Systems. Vol. II. Synoptic-Dynamic Meteorology in Midlatitudes, Oxford University Press, $608 \mathrm{pp}$.

Blunden, J., and D. S. Arndt, 2011: Analysis of freezing rain patterns in the south central United States: 1979-2009. Proc. 23rd Conf. on Climate Variability and Change, Seattle, WA, Amer. Meteor. Soc., 138. [Available online at https://ams.confex.com/ ams/91Annual/webprogram/Paper180091.html.]

Branick, M. L., 1997: A climatology of significant winter-type weather events in the contiguous United States, 1982-94. Wea. Forecasting, 12, 193-207, doi:10.1175/1520-0434(1997)012<0193: ACOSWT $>2.0 . \mathrm{CO} ; 2$.

- 2001: Monthly and geographic distribution of heavy snow events in Oklahoma, 1951-2001. National Weather Service Forecast Office, Norman, OK. [Available online at http:// www.srh.noaa.gov/oun/?n=climate-heavysnow-study.]

Brock, F. V., K. C. Crawford, R. L. Elliott, G. W. Cuperus, S. J. Stadler, H. L. Johnson, and M. D. Eilts, 1995: The Oklahoma Mesonet: A technical overview. J. Atmos. Oceanic Technol., 12, 5-19, doi:10.1175/1520-0426(1995)012<0005:TOMATO>2.0.CO;2.

Casola, J. H., and J. M. Wallace, 2007: Identifying weather regimes in the wintertime $500-\mathrm{hPa}$ geopotential height field for the Pacific-North American sector using a limited-contour clustering technique. J. Appl. Meteor. Climatol., 46, 1619-1630, doi:10.1175/ JAM2564.1.

Changnon, S. A., 2003: Characteristics of ice storms in the United States. J. Appl. Meteor., 42, 630-639, doi:10.1175/ 1520-0450(2003)042<0630:COISIT >2.0.CO;2.

— , and T. R. Karl, 2003: Temporal and spatial variations of freezing rain in the contiguous United States. J. Appl. Meteor., 42, 1302-1316, doi:10.1175/1520-0450(2003)042<1302: TASVOF $>2.0 . \mathrm{CO} ; 2$.

, D. Changnon, and T. R. Karl, 2006: Temporal and spatial characteristics of snowstorms in the contiguous United States. J. Appl. Meteor. Climatol., 45, 1141-1155, doi:10.1175/ JAM2395.1. 
Cortinas, J. V., Jr., B. C. Bernstein, C. C. Robbins, and J. W. Strapp, 2004: An analysis of freezing rain, freezing drizzle, and ice pellets across the United States and Canada: 1976-90. Wea. Forecasting, 19, 377-390, doi:10.1175/1520-0434(2004)019<0377: AAOFRF $>2.0 . \mathrm{CO} ; 2$.

Czys, R. R., R. W. Scott, K. C. Tang, R. W. Przybylinski, and M. E. Sabones, 1996: A physically based, nondimensional parameter for discriminating between locations of freezing rain and ice pellets. Wea. Forecasting, 11, 591-598, doi:10.1175/ 1520-0434(1996)011<0591:APBNPF>2.0.CO;2.

Grout, T., H. Yang, J. Basara, B. Balasundaram, Z. Kong, and T. S. Bukkapatnam, 2012: Significant winter weather events and associated socioeconomic impacts (federal aid expenditures) across Oklahoma: 2000-10. Wea. Climate Soc., 4, 48-58, doi:10.1175/WCAS-D-11-00057.1.

Hanesiak, J. M., and R. E. Stewart, 1995: The mesoscale and microscale structure of a severe ice pellet storm. Mon. Wea. Rev., 123, 3144-3162, doi:10.1175/1520-0493(1995)123<3144: TMAMSO $>2.0 . \mathrm{CO} ; 2$.

Harman, H. H., 1976: Modern Factor Analysis. University of Chicago Press, 487 pp.

Higgins, R. W., V. E. Kousky, and P. Xie, 2011: Extreme precipitation events in the south-central United States during May and June 2010: Historical perspective, role of ESNO, and trends. J. Hydrometeor., 12, 1056-1070, doi:10.1175/ JHM-D-10-05039.1.

Horel, J. D., 1981: A rotated principal component analysis of the interannual variability of the Northern Hemisphere $500 \mathrm{mb}$ height field. Mon. Wea. Rev., 109, 2080-2092, doi:10.1175/ 1520-0493(1981)109<2080:ARPCAO>2.0.CO;2.

Huffman, G. J., and G. A. Norman, 1988: The supercooled warm rain process and the specification of freezing precipitation. Mon. Wea. Rev., 116, 2172-2182, doi:10.1175/1520-0493(1988)116<2172: TSWRPA $>2.0 . \mathrm{CO} ; 2$.

Kalnay, E., and Coauthors, 1996: The NCEP/NCAR 40-Year Reanalysis. Bull. Amer. Meteor. Soc., 77, 437-471, doi:10.1175/ 1520-0477(1996)077<0437:TNYRP $>2.0$. CO;2.

Kennedy, A. D., X. Dong, B. Xi, S. Xie, Y. Zhang, and Y. Chen, 2011: A comparison of MERRA and NARR reanalyses with the DOE ARM SGP data. J. Climate, 24, 4541-4557, doi:10.1175/2011JCLI3978.1.

Lackmann, G., 2011: Midlatitude Synoptic Meteorology: Dynamics, Analysis and Forecasting. Amer. Meteor. Soc., 345 pp.

McPherson, R. A., and Coauthors, 2007: Statewide monitoring of the mesoscale environment: A technical update on the Oklahoma Mesonet. J. Atmos. Oceanic Technol., 24, 301-321, doi:10.1175/JTECH1976.1.

Mesinger, F., and Coauthors, 2006: North American Regional Reanalysis. Bull. Amer. Meteor. Soc., 87, 343-360, doi:10.1175/ BAMS-87-3-343.

Mullens, E. D., 2014: Moisture and thermal characteristics of Southern Plains ice storms: Insights from a regional synoptic climatology and high-resolution WRF-ARW sensitivity study. Ph.D. dissertation, University of Oklahoma, 342 pp. [Available online at https://shareok.org/handle/11244/10498.]

, L. M. Leslie, and P. J. Lamb, 2016: Impacts of Gulf of Mexico SST anomalies on southern plains freezing precipitation: ARW sensitivity study of the 28-30 January 2010 winter storm. J. Appl. Meteor. Climatol., 55, 119-143, doi:10.1175/ JAMC-D-14-0289.1.

North, G. R., T. L. Bell, R. F. Calahan, and F. J. Moeng, 1982: Sampling errors in the estimation of empirical orthogonal functions. Mon. Wea. Rev., 110, 699-706, doi:10.1175/ 1520-0493(1982)110<0699:SEITEO > 2.0.CO;2.

Rauber, R. M., M. K. Ramamurthy, and A. Tokay, 1994: Synoptic and mesoscale structure of a severe freezing rain event: The St. Valentine's Day ice storm. Wea. Forecasting, 9, 183-208, doi:10.1175/1520-0434(1994)009<0183:SAMSOA > 2.0.CO;2.

, L. Olthoff, M. Ramamurthy, and K. Kunkel, 2000: The relative importance of warm rain and melting processes in freezing precipitation events. J. Appl. Meteor., 39, 1185-1195, doi:10.1175/ 1520-0450(2000)039<1185:TRIOWR > 2.0.CO;2.

,,--- , D. Miller, and K. Kunkel, 2001: A synoptic weather pattern and sounding-based climatology of freezing precipitation in the United States east of the Rocky Mountains. J. Appl. Meteor., 40, 1724-1747, doi:10.1175/ 1520-0450(2001)040<1724:ASWPAS > 2.0.CO;2.

Ressler, G. M., S. M. Milrad, E. H. Atallah, and J. R. Gyakum, 2012: Synoptic-scale analysis of freezing rain events in Montreal, Quebec, Canada. Wea. Forecasting, 27, 362-378, doi:10.1175/ WAF-D-11-00071.1.

Richman, M. B., 1986: Rotation of principal components. J. Climatol., 6, 293-335, doi:10.1002/joc.3370060305.

_- and P. J. Lamb, 1985: Climatic pattern analysis of 3- and 7-day summer rainfall in the central United States: Some methodological considerations and a regionalization. J. Climate Appl. Meteor., 24, 1325-1343, doi:10.1175/1520-0450(1985)024<1325: CPAOTA $>2.0 . \mathrm{CO} ; 2$.

Riehl, H., and Coauthors, 1952: Forecasting in Middle Latitudes. Meteor. Monogr., No. 5, Amer. Meteor. Soc., 80 pp.

Robbins, C., and J. V. Cortinas Jr., 2002: Local and synoptic environments associated with freezing rain in the contiguous United States. Wea. Forecasting, 17, 47-65, doi:10.1175/ 1520-0434(2002)017<0047:LASEAW>2.0.CO;2.

Sanders, K. J., C. M. Gravelle, J. P. Gagen, and C. E. Graves, 2013: Characteristics of major ice storms in the central United States. J. Oper. Meteor., 1, 100-113, doi:10.15191/ nwajom.2013.0110.

Stewart, R. E., 1985: Precipitation types in winter storms. Pure Appl. Geophys., 123, 597-609, doi:10.1007/BF00877456.

— storms. Mon. Wea. Rev., 115, 1270-1279, doi:10.1175/ 1520-0493(1987)115<1270:FPIWS>2.0.CO;2.

Stokes, G. M., and S. E. Schwartz, 1994: The Atmospheric Radiation Measurement (ARM) program: Programmatic background and design of the Cloud and Radiation Test Bed. Bull. Amer. Meteor. Soc., 75, 1201-1221, doi:10.1175/ 1520-0477(1994)075<1201:TARMPP>2.0.CO;2.

Wang, P. Y., J. E. Martin, J. D. Locatelli, and P. V. Hobbs, 1995: Structure and evolution of winter cyclones in the United States and their effects on the distribution of precipitation. Part II: Arctic fronts. Mon. Wea. Rev., 123, 1328-1344, doi:10.1175/ 1520-0493(1995)123<1328:SAEOWC > 2.0.CO;2.

Zerr, R. J., 1997: Freezing rain: An observational and theoretical study. J. Appl. Meteor., 36, 1647-1661, doi:10.1175/ 1520-0450(1997)036<1647:FRAOAT>2.0.CO;2. 